\title{
Interaction of two adjacent structures coupled by inerter-based system considering soil conditions
}

\author{
Zhipeng Zhao ${ }^{\mathrm{a}, \mathrm{b}}$, Qingjun Chen ${ }^{\mathrm{a}, \mathrm{b}}$, Ruifu Zhang ${ }^{\mathrm{b}}$, Yiyao Jiang ${ }^{\mathrm{b}}$, Yuying Xia ${ }^{\mathrm{c}}$ \\ ${ }^{a}$ State Key Laboratory of Disaster Reduction in Civil Engineering, Tongji University, Shanghai 200092, China \\ ${ }^{b}$ Department of Disaster Mitigation for Structures, Tongji University, Shanghai 200092, China \\ ${ }^{c}$ Swansea University, College of Engineering, Fabian Way, SA1 8EN, Swansea, UK
}

\begin{abstract}
The inerter-based systems have proven to be effective for vibration control of adjacent structures. The interaction through the soil medium between adjacent structures in urban areas is generally accepted. However, existing studies concerning the inerter-based adjacent structures are primarily based on the assumption of a fixed base, without considering the inevitable interaction. To address this issue, this study incorporated the soil effects into the theoretical analysis of adjacent structures interconnected by an inerter system, and correspondingly develops an optimal design framework for such system. Employing a classic discrete model for structures and soil, the interaction behavior between inerter-based adjacent structures and soil was extensively studied in a comparative analysis. Based on the revealed interaction phenomena, the need for considering the soil condition in the design of an inerter system for adjacent structures was addressed, and a performance-demand-based optimal design framework was developed. The results indicated that for inerter-based adjacent structures spaced closely, the coupled interaction effect of soil and structure requires careful consideration, especially in soft soil conditions. Owing to the soil effects, the inerter system exhibited a weakened effectiveness for displacement reduction. A larger inner deformation of the inerter system is required to meet the demand for energy dissipation. With consideration of the soil condition, the proposed design method can satisfy the pre-specified target displacement demands for adjacent structures, simultaneously optimizing the control cost as an economical solution.
\end{abstract}

Keywords: inerter; soil-structure interaction; structure-soil-structure interaction; adjacent structures; optimal design.

${ }^{*}$ Corresponding author.

Email address: zhangruifu@tongji.edu.cn 


\section{Introduction}

Interconnecting adjacent structures with supplemental control devices is a practical and effective approach to avoid unexpected pounding and reduce the dynamic responses simultaneously. The relevant methodologies and energy dissipating systems have been analyzed theoretically [1,2] and experimentally [3, 4]. The approach proposed by Westermo [5] used a hinged link to couple the adjacent structures for pounding reduction. Zhang and $\mathrm{Xu}$ [6] investigated the seismic performance of adjacent structures linked by discrete viscoelastic dampers (simulated by linear damping and stiffness elements in parallel). Xu et al. [4] further experimentally analyzed the dynamic characteristic and harmonic response of a pair of adjacent structures coupled by a fluid viscous damper (simulated by linear damping and stiffness elements in series). Later, Ni et al. [7] developed a measure for analyzing the random seismic response of two adjacent structures interconnected by a nonlinear hysteretic damper. Basili and De Angelis [8] introduced a reduced model for the optimal design of two adjacent structures connected by a hysteretic damper. Adopting a recently developed new device, i.e., the inerter element, Basili, De Angelis, and Pietrosanti [9] proposed a structural configuration that linked adjacent structures by a springdashpot-inerter system (SDIS), and then analyzed its modal characteristic and dynamic responses; however, any effects due to the spatial variation of the ground or to the soil-structure interaction (SSI) were neglected.

The mentioned inerter is essentially a two-terminal mechanical element [10], ideally generating an inertia force proportional to the relative acceleration between its two terminals and an apparent mass called inertance with negligible physical mass, which is named as mass enhancement mechanism. In the 1970s, Kawamata [11] developed a liquid mass pump by utilizing the inertial resistance of the flowing liquid, which represented the origin of the use of a two-terminal inertial element in the field of civil engineering. Subsequently, Ikago et al. proposed a two-terminal inertial system, the tuned viscous mass damper, which explicitly used the inertial mass enhancement and damping enhancement effects for the first time [10]. Zhang et al. [12] derived a closed-form damping enhancement equation to establish the theoretical basis of the damping enhancement effect. Owing to its unique advantages of inertial mass enhancement and damping enhancement effects, the inerter exhibits huge potential in the realization of "lightweight" tuned-like control devices [13-17], where the role of the tuned mass is partially replaced by the inerter. Compared with the conventional tuned-type dampers, much less tuned mass is required for the inerter-based tuned-like devices to provide the same control effect. Moreover, the inerter allows developing an energy dissipating device with improved effectiveness [18] and efficiency. Among the proposed inerter-based passive vibration-suppression configurations, the commonly considered ones include the tuned viscous mass damper [10], tuned inerter damper [19], tuned mass damper inerter [20], and SDIS [21]. As for the SDIS considered in this study, $\mathrm{Hu}$ et al. [22] analyzed the optimization problem for SDIS-based isolators through the fixed-point theory. Jiang et al. [23] derived the analytical design formulae of the SDIS for the vibration mitigation of a storage tank, especially revealing the benefit of the inerter to suppress sloshing responses.

As summarized, the extant studies corresponding to inerter-based structures were performed based on the assumption of a fixed-base condition, i.e., the impact of the SSI on the seismic performance and vibration mitigation effect of inerter- 
based structures was neglected. However, the arbitrary assumption of the fixed-base support is valid only for the case of a structure standing on rock or soil with high stiffness [24]. As for the soft soil condition, the interaction between soil and structure results in a decrease of the fundamental frequency and a modification of the energy dissipation mechanism [2528]. Dealing with the SSI impact on the inerter-based system, Chen et al. [29] addressed the need of incorporating the SSI effect into the analysis and parameter determination of the inerter-based system, and correspondingly proposed an optimal design method. This study mainly dealt with the assessment of the seismic performance of a single structure far from the other surrounding structures. However, in the case of buildings located in close proximity, the interaction and coupling effect of the adjacent structures via soil is inevitable. The studies concerning the structure-soil-structure interaction (SSSI) have resulted in some special considerations, as summarized in [30], for a complete review of the issue. It has been concluded that to obtain satisfactory results for a precise seismic analysis, the SSSI effects cannot be neglected. Several studies have been conducted to assess the influence of the coupling effect in SSSI phenomena on the seismic performance, predominantly by means of experiments [31,32] and the finite element [33] and reduced-order model [34-36] methods. Among these, the reduced-order model saves time and its clear and concise mechanical configuration is particularly effective for the parametric analysis to investigate how the structural performance is affected by the SSSI effect and for appreciating the basic features of the problem. Alexander et al. [35] conducted a systematic study to propose a simple discrete model for the interaction of adjacent structures. The higher mode interaction between the structures [36] and nonlinear behavior of the soil [33] were later evaluated. Referring to the previous studies, it has been found that the soil effect (including the uncoupled SSI and coupled SSSI) has a significant influence on the dynamic characteristics of adjacent structures, which successively affects the structural responses and performance of the interconnection devices. Especially for the adjacent structures-SDIS (AS-SDIS), the SDIS is a displacement-, velocity-, and acceleration-dependent control device and its vibration mitigation effect is closely related to the structural dynamic characteristics. Hence, the interaction behavior of the AS-SDIS with an underlying soil medium should be analyzed and incorporated in its design.

To this end, this study analyzed the interaction behavior between the AS-SDIS and the soil medium by incorporating the coupled SSSI and uncoupled SSI effects into the analysis through the simple and classic discrete models for structures and soil. A performance-demand-based optimal design method was developed for this interaction system with consideration of the soil condition. The stochastic analysis model for this interaction system was established and the corresponding results were used in an extensive parametric analysis. Moreover, the seismic behavior of the SDIS and energy dissipation balance of the interaction system were further evaluated under the excitation of various earthquakes. Finally, the effectiveness of the proposed design method was verified through a series of cases.

\section{Theoretical analysis}

\subsection{Spring-dashpot-inerter system model}

Fig. 1 shows a schematic diagram of the SDIS, comprising three mechanical elements: a spring element $\left(k_{d}\right)$, a dashpot element $\left(c_{d}\right)$, and an inerter element $\left(m_{i n}\right)$. All the three elements are placed in parallel between the same two 
terminals to form the system. For the two adjacent structures, the SDIS has proven to be effective for vibration control [9, 37], and it is simultaneously the simplest way to utilize an inerter as a linking element without introducing an extra degree of freedom (DOF) in the AS-SDIS.

The inerter mentioned above is a two-terminal mechanical element and can ideally generate an inertia force proportional to the relative acceleration of the two terminals and inertance [38-41]. The significant feature of the inerter is that the inertance can be thousands of times its physical mass [10, 42], which is termed as the mass enhancement effect. Compared with the primary structure, even though a large inertance is designed in the SDIS, its physical mass is negligible. Thus, the idea of utilizing the inerter as a coupling element aims to improve the structural performance with almost no additional gravitational mass. Fig. 1 shows the mechanical model of the SDIS, where $u_{\text {left }}$ and $u_{\text {right }}$ denote the displacements of the two terminals. The output force of the SDIS, $F_{S D I S}$, can be calculated as follows:

$$
F_{\text {SDIS }}=k_{d}\left(u_{\text {right }}-u_{\text {left }}\right)+c_{d}\left(v_{\text {right }}-v_{\text {left }}\right)+m_{\text {in }}\left(a_{\text {right }}-a_{\text {left }}\right),
$$

where $v$ and $a$ signify the velocity and acceleration responses.

Unlike the typical coupling elements, such as a spring and a dashpot, the SDIS is a displacement-, velocity-, and acceleration-dependent control system. Its force (Eq. (1)) is dependent on the displacement, velocity, and acceleration responses between the adjacent structures, and it is substantially dependent on the dynamic characteristics of the adjacent structures [9]. If the dynamic characteristics of the adjacent structures change (for example, when the fixed-base assumptions are inapplicable owing to the SSI effect), the behavior of the SDIS for dynamic response mitigation (reflected by $\left.F_{S D I S}\right)$ cannot be expected. Furthermore, considering the possibility of interaction and the coupling of adjacent structures through the soil beneath the structures, the vibration mitigation effect of the SDIS can also be influenced by the SSSI effect. Focusing on this issue, the beneficial or adverse effect of the SSSI and uncoupled SSI on the dynamic performance of the AS-SDIS will be analyzed in the following sections.

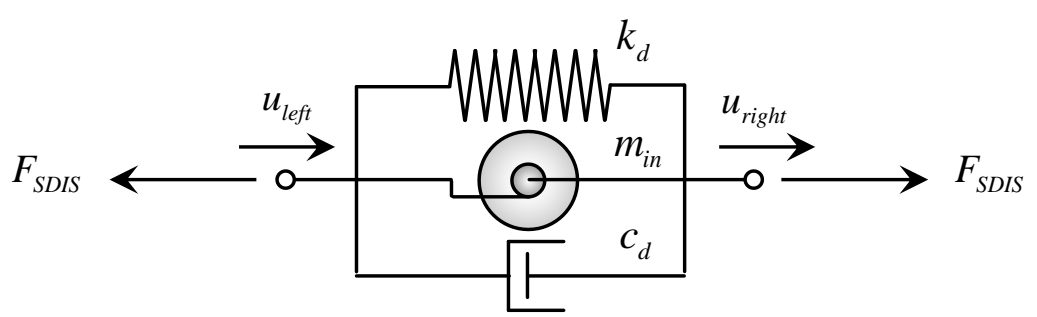

Fig. 1. Schematic of mechanical model of the SDIS.

\subsection{AS-SDIS model considering soil and structure effects}

Considering a pair of 1-DOF primary structures (Fig. 2 (a)), each structure is defined by structural stiffness $k_{i}$, mass $m_{i}$, and structural height $h_{i}$. In this study, unless otherwise stated, $i=1,2$. To impose the soil and structure effects onto the modeling procedure, an extra rotational DOF is introduced at the bottom of each structure (Fig. 2 (b)) and an interstructure spring $k_{\theta}$ is employed to couple the two adjacent structures. By means of this rotational DOF, the rocking modes of the AS-SDIS can be properly considered.

The selected discrete SSSI model has been validated through a comparison with the classic finite element method [35] and developed for more complicated adjacent models $[33,36]$. Owing to its advantages of saving of time and reasonable 
accuracy for practical engineering, the discrete model is used in this study to theoretically investigate the soil and structure effects on the AS-SDIS from the perspective of a physical basis [35]. A time-delayed ground excitation is neglected in this model. Thus, wave passage effects and spatially heterogeneous ground displacements are not taken into account in the present work to avoid the complexity of the AS-SDIS system $[35,36]$.

The foundation is characterized by the foundation width $B_{i}$, foundation mass-radius of gyration $r_{i}$, rotational soil spring $k_{s i}$, and soil/foundation mass underneath the structure $m_{s i} . B_{z}$ denotes the inter-structure distance. The ground acceleration $a_{g}$ is applied at both foundations without considering the wave passage effect and building pounding between the adjacent structures. Owing to the soil effect, each structure of the AS-SDIS is represented by a simplified model with 2-DOFs, which refer to one rotational DOF at the foundation level, $\left(\theta_{i}\right)$ and one translational DOF $\left(u_{i}\right)$.

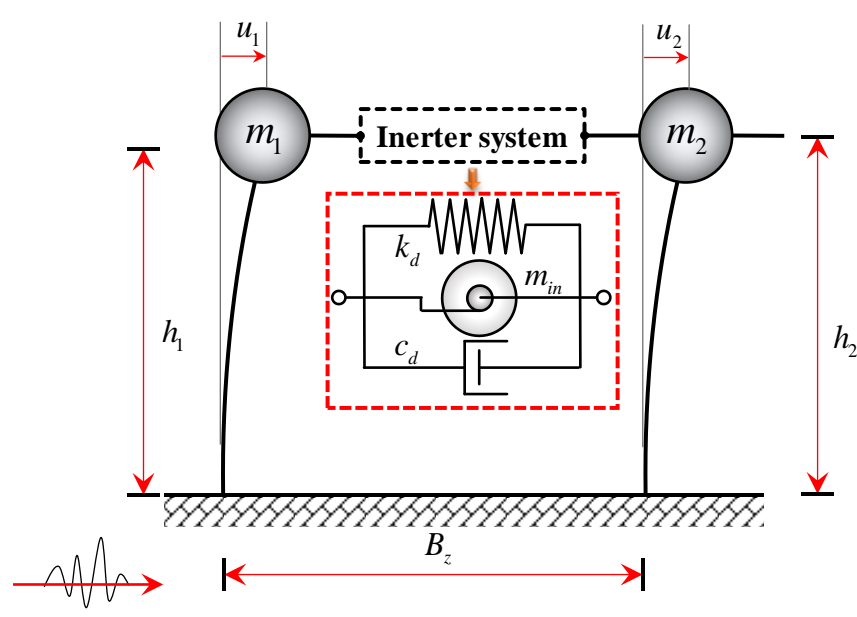

(a) AS-SDIS model on fixed base

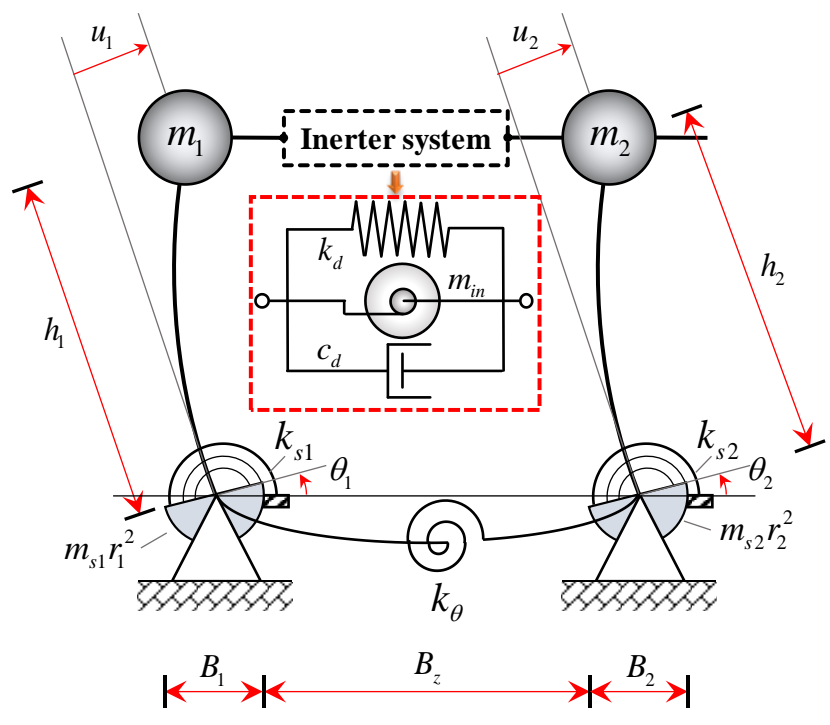

(b) AS-SDIS model considering soil and structure effects

Fig. 2. Schematics of mechanical models of the AS-SDIS considered in this study.

\subsection{Stochastic analysis}

With reference to Fig. 2, in this section, the governing equation of motion is established and the dynamic responses of the AS-SDIS founded on an elastic-flexible soil are analyzed. In the case of uncontrolled adjacent structures (Fig. 2 (a), with the symbols denoting one rotational DOF at the foundation level $\left(\theta_{i}\right)$ and one translational DOF $\left.\left(u_{i}\right)\right)$, the basic motion equations of the adjacent structures on the flexible soil subject to the ground excitation $a_{g}$ are established as

$$
\mathbf{M}_{\mathbf{u}} \mathbf{a}+\mathbf{C}_{\mathbf{u}} \mathbf{v}+\mathbf{K}_{\mathbf{u}} \mathbf{u}=\mathbf{P} a_{g}
$$

where $\mathbf{M}_{\mathbf{u}}, \mathbf{C}_{\mathbf{u}}$, and $\mathbf{K}_{\mathbf{u}}$ represent the mass, damping, and stiffness matrixes of the uncontrolled adjacent structures, respectively. The above matrixes are expressed in detail as 
$\mathbf{M}_{\mathbf{u}}=\left[\begin{array}{cccc}m_{1} & -m_{1} h_{1} & 0 & 0 \\ -m_{1} h_{1} & m_{1} h_{1}^{2}+m_{s 1} r_{1}^{2} & 0 & 0 \\ 0 & 0 & m_{2} & -m_{2} h_{2} \\ 0 & 0 & -m_{2} h_{2} & m_{2} h_{2}^{2}+m_{s 2} r_{2}^{2}\end{array}\right], \mathbf{K}_{\mathbf{u}}=\left[\begin{array}{cccc}k_{1} & 0 & 0 & 0 \\ 0 & k_{s 1}+k_{\theta} & 0 & -k_{\theta} \\ 0 & 0 & k_{2} & 0 \\ 0 & -k_{\theta} & 0 & k_{s 2}+k_{\theta}\end{array}\right], \mathbf{C}_{\mathbf{u}}=\mathbf{M}_{\mathbf{u}}\left(\sum_{n=1}^{4} \frac{2 \zeta \omega_{n}}{\phi_{n}^{T} \mathbf{M}_{\mathbf{u}} \phi_{n}} \phi_{n} \phi_{n}^{T}\right) \mathbf{M}_{\mathbf{u}}$

where the linear viscous damping matrix $\mathbf{C}_{\mathbf{u}}$ is defined in the condition that each mode is damping at $\zeta$ of the critical damping. $\omega_{n}$ and $\phi_{n}$ are, respectively, the natural frequency and eigenvector for the mode $n$. $\mathbf{u}$ and $\mathbf{P}$ represent the displacement and excitation vectors, respectively expressed as $\mathbf{u}=\left[u_{1}, \theta_{1}, u_{2}, \theta_{2}\right]^{T}$ and $\mathbf{P}=\left[-m_{1}, m_{1} h_{1},-m_{1}, m_{2} h_{2}\right]^{T}$. $\mathbf{v}=\left[v_{1}, v_{\theta_{1}}, v_{2}, v_{\theta_{2}}\right]^{T}$ and $\mathbf{a}=\left[a_{1}, a_{\theta_{1}}, a_{2}, a_{\theta_{2}}\right]^{T}$ denote respectively the velocity and acceleration vectors that can be obtained by taking the first and second derivative of $\mathbf{u}$ with respect to the time. In Eq. (3), the motions of the AS-SDIS are expressed in the mechanical parameter term with a relative large number of system parameters. The next step is to normalize the system and simplify these parameters into a smaller set.

For two adjacent structures, this study approximates the natural period of the fixed-base structure as $T=N / 10$ (where $N$ denotes the number of stories [43]). In the assumption of the average height of each story $h_{\text {ave }}$, the following expressions can be obtained: the structural height $h_{i}=N_{i} h_{\text {ave }}$, and the corresponding fundamental frequency of the structures $\omega_{s i}=20 \pi h_{\text {ave }} / h_{i}$. Moreover, prior to developing the analytical formulations, other assumptions to limit the scope of the subsequent analysis are listed as follows [44]: (a) the adjacent structures are founded on the same soil profile, so that $k_{s 1}=k_{s 2}$; (b) both structures are shaped as a square plan area of $B^{2}$, where $r_{1}=r_{2}=0.33 B$; (c) both building structures have the same equivalent mass density, $\rho_{b} ;$ (d) the structures are characterized with different heights $h_{i}$.

To establish the relationship of the structural mechanical parameters with the geometry information, the suggestion given by Newmark and Rosenblueth [45] is used here: the soil/foundation mass underneath the structure is $m_{s i}=0.35 B^{3} \rho_{s}$, where $\rho_{s}$ denotes the mass density of the soil. In addition, the mass of the adjacent structures can be calculated as $m_{i}=\rho_{b} B^{3} h_{i}$. In the case of the SSSI model, empirical equations are employed to determine the value of $k_{s i}$ and $k_{\theta}$ based on the rotational spring $k_{s}$ as $[35,36]$

$$
k_{s}=\frac{0.5 G_{s} B^{3}}{(1-v)}, k_{s i}=\left(1+\frac{0.5}{(1+z)^{3}}\right) k_{s}, k_{\theta}=\left(-\frac{0.25}{(1+z)^{3}}\right) k_{s 1},
$$

where $G_{s}=\rho_{s} V_{s}^{2}$ and $v$ denote the elastic shear modulus and the Poisson's ratio of the soil, respectively. $z=B_{z} / B$ is the inter-structure distance ratio, and $V_{s}$ represents the shear wave velocity of the soil. Based on the above introduction, the complete problem of adjacent structures considering the SSSI effect is described in non-dimensional form through the parameters summarized in Table 1. Finally, the normalized dynamic input excitation $\bar{u}_{g}$, responses $\bar{u}_{i}, \bar{\theta}_{i}$, and time-scale $\tau$ are given as

$$
\bar{u}_{i}=u_{i} / r_{i}, \bar{u}_{\theta i}=\theta_{i}, \bar{a}_{g}=a_{g} / r_{1}, \tau=\omega_{s 1} t
$$


Table 1. Parameters of the adjacent structures with consideration of soil and structure effects.

\begin{tabular}{lll}
\hline Parameters & Definition & Description \\
\hline \multirow{3}{*}{ Geometric parameters } & $s=h_{1} / B$ & Aspect ratio \\
& $\varepsilon=h_{2} / h_{1}$ & Height ratio \\
& $z=B_{z} / B$ & Inter-structure distance ratio \\
\hline & $\bar{V}_{s}=V_{s} / 1000$ & Normalized wave shear velocity \\
Soil parameters & $\alpha_{1}=0.35 \rho_{s} / \rho_{b}$ & Soil mass ratio \\
& $\alpha_{2}=327.5 /(1-v)$ & Soil frequency ratio $\left(h_{\text {ave }}=3.2 \mathrm{~m}[35]\right)$ \\
& $\beta_{s}=k_{s 1} / k_{s}$ & Rotational soil stiffness ratio \\
& $\beta_{\theta}=k_{\theta} / k_{s 1}$ & Inter-structure stiffness ratio \\
\hline
\end{tabular}

Substituting the given system parameters into the governing equation of motion, Eq. (3) can be re-expressed in the non-dimensional form as follows:

$$
\begin{aligned}
& \overline{\mathbf{M}}_{\mathbf{u}}=\left[\begin{array}{cccc}
1 & -3 s & 0 & 0 \\
-3 s & 9 s^{2}+\alpha_{1} s^{-1} & 0 & 0 \\
0 & 0 & \varepsilon & -3 \varepsilon^{2} s \\
0 & 0 & -3 \varepsilon^{2} s & 9 \varepsilon^{3} s^{2}+\alpha_{1} s^{-1}
\end{array}\right], \overline{\mathbf{K}}_{\mathbf{u}}=\left[\begin{array}{cccc}
1 & 0 & 0 & 0 \\
0 & \alpha_{1} \alpha_{2} \beta_{s} s \bar{V}_{s}^{2}\left(1+\beta_{\theta}\right) & 0 & -\alpha_{1} \alpha_{2} \beta_{s} \beta_{\theta} s \bar{V}_{s}^{2} \\
0 & 0 & \varepsilon^{-1} & 0 \\
0 & -\alpha_{1} \alpha_{2} \beta_{s} \beta_{\theta} s \bar{V}_{s}^{2} & 0 & \alpha_{1} \alpha_{2} \beta_{s} s \bar{V}_{s}^{2}\left(1+\beta_{\theta}\right)
\end{array}\right], \\
& \overline{\mathbf{C}}_{\mathbf{u}}=\overline{\mathbf{M}}_{\mathbf{u}}\left(\sum_{n=1}^{4} \frac{2 \zeta \omega_{n}}{\phi_{n}^{T} \overline{\mathbf{M}}_{\mathbf{u}} \phi_{n}} \phi_{n} \phi_{n}^{T}\right) \overline{\mathbf{M}}_{\mathbf{u}}, \overline{\mathbf{P}}=\left[\begin{array}{c}
-1 \\
3 s \\
-\varepsilon \\
3 \varepsilon^{2} s
\end{array}\right], \overline{\mathbf{u}}=\left[\begin{array}{c}
\bar{u}_{1} \\
\bar{u}_{\theta 1} \\
\bar{u}_{2} \\
\bar{u}_{\theta 2}
\end{array}\right] .
\end{aligned}
$$

In the case of the AS-SDIS, the non-dimensional parameters of SDIS are introduced in Table 2. Incorporating the SDIS into adjacent structures, the excitation vector $\overline{\mathbf{P}}$ is not changed, because no extra seismic force is introduced into the AS-SDIS owing to the mass enhancement effect. The basic equation of AS-SDIS can be developed, based on Eqs. (2), (6), and (7), as follows:

$$
\overline{\mathbf{M}} \overline{\mathbf{u}}+\overline{\mathbf{C}} \overline{\mathbf{v}}+\overline{\mathbf{K}} \overline{\mathbf{a}}=\overline{\mathbf{P}} \bar{a}_{g}
$$

where,

$$
\begin{gathered}
\overline{\mathbf{M}}=\overline{\mathbf{M}}_{\mathbf{u}}+\mu \overline{\mathbf{M}}_{\mathrm{AS}}, \overline{\mathbf{K}}=\overline{\mathbf{K}}_{\mathbf{u}}+\kappa \overline{\mathbf{M}}_{\mathrm{AS}}, \overline{\mathbf{C}}=\overline{\mathbf{C}}_{\mathbf{u}}+2 \xi \overline{\mathbf{M}}_{\mathrm{AS}}, \\
\overline{\mathbf{M}}_{\mathrm{AS}}=\left[\begin{array}{cccc}
1 & -3 s & -1 & 3 \varepsilon s \\
-3 \mu s & 9 s^{2} & 3 s & -9 \varepsilon s^{2} \\
-1 & 3 s & 1 & -3 \varepsilon s \\
3 \varepsilon s & -9 \varepsilon s^{2} & -3 \varepsilon s & 9 \varepsilon^{2} s^{2}
\end{array}\right]
\end{gathered}
$$

$\overline{\mathbf{M}}_{\mathrm{AS}}$ is the connection matrix of adjacent structures. $\overline{\mathbf{v}}$ and $\overline{\mathbf{a}}$ are normalized velocity and acceleration vectors.

Table 2. Parameters of the SDIS.

\begin{tabular}{lll}
\hline Parameters & Definition & Description \\
\hline & $\mu=m_{i n} / m_{1}$ & Inertance-mass ratio \\
Inerter system parameters & $\kappa=k_{d} / k_{1}$ & Stiffness ratio \\
& $\xi=c_{d} / 2 \sqrt{k_{1} m_{1}}$ & Damping ratio \\
\hline
\end{tabular}


Considering the excitation as a stochastic process, the stochastic analysis needs to be conducted on the basis of the complex mode and pseudo-excitation method [46]. Supposing a harmonic excitation with circular frequency $\omega$ and power spectral density $S_{g}(\omega)$, the excitation can be expressed as $a_{g}(t)=\sqrt{S_{g}(\omega)} e^{\mathrm{i} \omega t}$. The power spectral density matrix of the displacement response of the two adjacent structures $\left(\mathbf{S}_{\mathbf{u}}(\omega)\right)$ can be calculated $[47,48]$.

\section{Interaction between soil and AS-SDIS}

Referring to the established AS-SDIS model and the corresponding stochastic analysis, a parametric analysis is conducted in this section to investigate the interaction behavior between the adjacent structures and the soil. Based on the parametric analysis results, the need for incorporating the soil effect into the SDIS design is addressed.

\subsection{Defining performance measures}

As a measure of the difference between the dynamic responses of the adjacent structures in the fixed-base state and when influenced by the soil and structure effects, in this section, the following performance measures are defined as

$$
U_{i}=u_{i}-\theta_{i} H_{i}, A_{i}=a_{i}+a_{g}-a_{\theta_{i}} H_{i},
$$

where $U_{i}$ and $A_{i}$ are, respectively, the relative (sway + rotational) displacement and total (sway + rotational + ground) acceleration for structure $i$. The response mitigation ratio in terms of displacement $\alpha_{U_{i}, \text { SSSI }}$ and acceleration $\alpha_{A_{i}, \text { SSSI }}$ between the adjacent structures in the coupled SSSI state is defined as

$$
\alpha_{U_{i}, \text { SSSI }}=\frac{\left[\mathrm{E}\left(U_{i}\right)\right]_{\text {SSSI }}}{\left[\mathrm{E}\left(U_{i}\right)\right]_{\text {Uncontrolled }}}, \alpha_{A_{i}, \text { SSSI }}=\frac{\left[\mathrm{E}\left(A_{i}\right)\right]_{\text {SSSI }}}{\left[\mathrm{E}\left(A_{i}\right)\right]_{\text {Uncontrolled }}},
$$

where the subscript 'Uncontrolled' indicates the adjacent structures founded on the fixed base without any control devices. In a similar way, the evaluation indices $\alpha_{U_{i}, \text { SSI }}, \quad \alpha_{U_{i}, \text { Fixed-base }}, \quad \alpha_{A_{i}, \text { SSI }}$, and $\alpha_{U_{i}, \text { Fixed-base }}$ are given as

$$
\alpha_{U_{i}, \text { SSI }}=\frac{\left[\mathrm{E}\left(U_{i}\right)\right]_{\mathrm{SSI}}}{\left[\mathrm{E}\left(U_{i}\right)\right]_{\text {Uncontrolled }}}, \alpha_{A_{i}, \text { SSI }}=\frac{\left[\mathrm{E}\left(A_{i}\right)\right]_{\mathrm{SSI}}}{\left[\mathrm{E}\left(A_{i}\right)\right]_{\text {Uncontrolled }}}, \alpha_{U_{i}, \text { Fixed-base }}=\frac{\left[\mathrm{E}\left(U_{i}\right)\right]_{\text {Fixed-base }}}{\left[\mathrm{E}\left(U_{i}\right)\right]_{\text {Uncontrolled }}}, \alpha_{A_{i}, \text { Fixed-base }}=\frac{\left[\mathrm{E}\left(A_{i}\right)\right]_{\text {Fixed-base }}}{\left[\mathrm{E}\left(A_{i}\right)\right]_{\text {Uncontrolled }}} \text {. (12) }
$$

with the subscripts 'SSSI,' 'SSI,' and 'Fixed-base' standing for the adjacent structures under the assumption of SSI, SSSI, and fixed-base states. To obtain the seismic behavior of the uncoupled SSI system, the inter-structure spring is set to zero and $k_{s i}=k_{s}$ for simplification. This assumption is also the base for the comparative study of SSSI and SSI effects [35, 36]. The operator $\mathrm{E}$ denotes the root mean square operation based on the stochastic results, as follows:

$$
\mathrm{E}\left(U_{i}\right)=\left[\int_{-\infty}^{+\infty} \mathbf{S}_{U_{i}}(\omega) \mathrm{d} \omega\right]^{1 / 2} .
$$

Utilizing the introduced root mean square operation, the defined performance measures can deliver a statistic 
evaluation of the magnitude of the dynamic response difference between different assumptions, i.e., the fixed base, SSI, and coupled SSSI states. This measurement criterion is more robust than the evaluation based on the single peak response.

\subsection{Parametric analysis of soil effects on AS-SDIS}

Selecting the defined performance indices as the evaluating measures, this section conducts an extensive parametric analysis by considering the different conditions of the soil, inter-structure distance, and the two adjacent buildings.

\subsubsection{Influence of inter-structure distance ratio $z$}

As commonly known, the inter-structure distance between two adjacent buildings is a key factor in determining whether the SSSI effect is significant in the interaction between soil and structure, further resulting in a different performance of a structure founded on the flexible soil. In the exploration of how the structural performances $\left(\alpha_{U_{i}}\right.$ and $\alpha_{A_{i}}$ ) change with the inter-structure distance ratio $z$, we consider the cases of three soil conditions, i.e., soft, medium, and dense soil [36], and different sets of parameters of the adjacent structures' shapes [35], that is, $(s, \varepsilon)$ as $(0.5,1.25)$, $(2.0,1.25)$, and $(2.0,3.0)$. For the SDIS, $\mu, \kappa$, and $\xi$ are assumed as $0.3,0.1$, and 0.1 , which are selected as a typical example. The values of the soil parameters defined in Table 1 can be determined by the type of soil and reference [36]. The variation trends of $\alpha_{U_{i}}$ and $\alpha_{A_{i}}$ obtained in this section are not limited by the values of SDIS.

Selecting the typical results, Fig. 3 and Fig. 4 show the analysis results of $\alpha_{U_{i}}$ and $\alpha_{A_{i}}$ of two adjacent structures with $(s=0.5, \varepsilon=1.25)$ and $(s=2.0, \varepsilon=3.0)$ in the states of fixed base (dotted lines marled by triangles), with SSI effect (dotted lines with marks), and with coupled SSSI effect (bold lines with marks). For the structure founded on the flexible soil, the consideration of soil impact can be analogous to the insertion of an isolation system between the fixedbase and the rigid superstructure. Because of this isolation-like effect, the structural acceleration response is reduced, whereas the displacement responses are increased significantly, compared with the structure on the fixed base. As the soil condition changes from firm to soft, the soil stiffness becomes lower, and thus, the mentioned trends of displacement and acceleration become more significant. For two closely spaced adjacent structures, i.e., $z \quad$ is small $(z<1)$, the coupled SSSI is significant, which leads to a non-negligible difference of $\alpha_{U_{i}}$ and $\alpha_{A_{i}}$ compared with the structure influenced by an uncoupled SSI effect. As the adjacent structures move away from each other, the coupled effect is gradually attenuated and reduced to a negligible level. In conclusion, the SSI effect is definitely an important factor that should be properly considered for the analysis of the AS-SDIS. Especially for closely spaced adjacent structures, consideration of the coupled SSSI effect is required, and the reason will be addressed in the following discussion.

Fig. 3 shows the case where Structure 2 is $25 \%$ higher than the first one, and Structure 1 has an aspect ratio of 0.5 
$(s=0.5, \varepsilon=1.25)$. Incorporating the influence of soil with the AS-SDIS, the displacement responses are increased in comparison with those in the fixed-base state. This increase in the displacement responses can be attributed to the rotation of the foundation in the rocking DOF, which may further lead to unexpected pounding between the adjacent structures spaced at a small distance. Comparing the results of $\alpha_{U_{i}}$ and $\alpha_{A_{i}}$ in the SSI and SSSI cases, Structure 1 is significantly affected by the taller Structure 2 through the negative interaction effect of $k_{\theta}$ and the strengthened $k_{s i}$. An amplification of the displacement and acceleration responses is found (Fig. 3 (a) and (c)), especially in the case of a small inter-structure distance. In terms of Structure 2, which is placed near the lower Structure 1, there is a remarkable decrease in the displacement response influenced by the SSSI effect compared to that influenced by the SSI effect. The lower structure can be viewed as a tuned mass connected with the taller structure through the SDIS and inter-structure spring $k_{\theta}$, correspondingly resulting in this reduction.

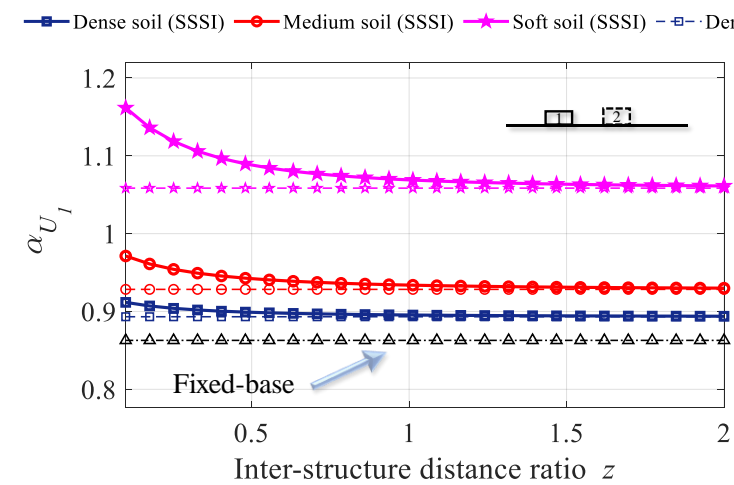

(a)

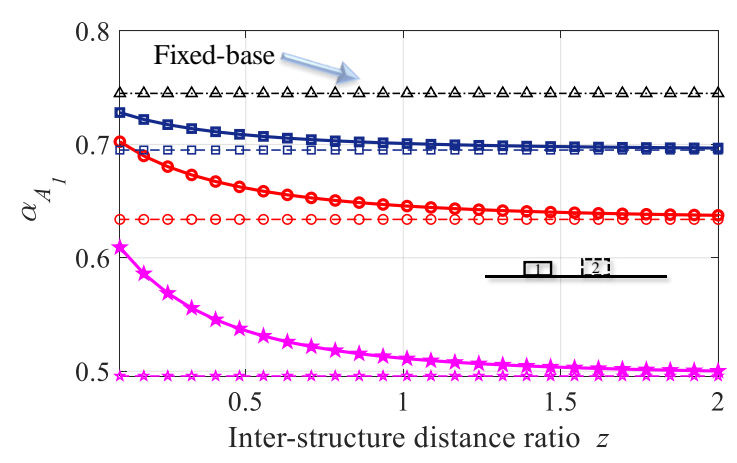

(c)

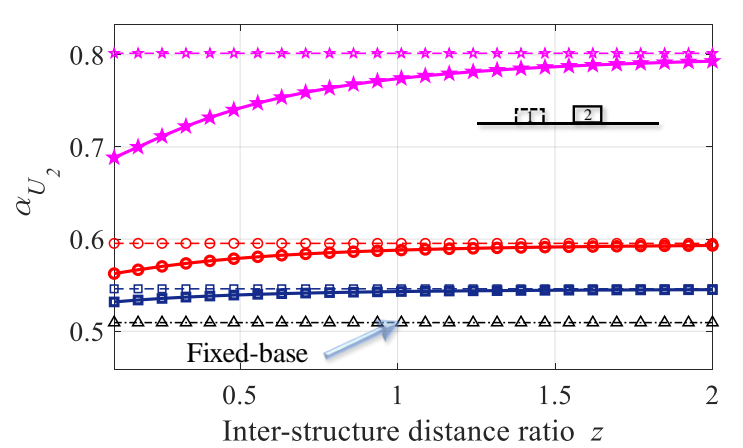

(b)

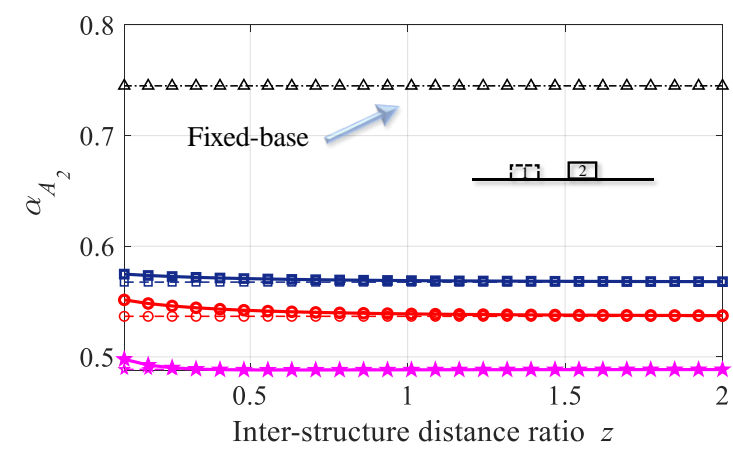

(d)

Fig. 3. Seismic response of AS-SDIS varied with the inter-structure distance ratio $z$ in the case of $(s=0.5, \varepsilon=1.25)$ : (a) displacement response mitigation ratio of Structure $1 \alpha_{U_{1}}$; (b) displacement response mitigation ratio of Structure 2 $\alpha_{U_{2}} ;$ (c) acceleration response mitigation ratio of Structure $1 \alpha_{A_{1}} ;(\mathrm{d})$ acceleration response mitigation ratio of Structure $2 \alpha_{A_{2}}$.

Fig. 4 shows the case where the second structure is two times higher than the first one, and Structure 1 has an aspect ratio of $2.0(s=2.0, \varepsilon=3.0)$. The variation trend of $\alpha_{U_{2}}$ and $\alpha_{A_{2}}$, on the whole, is the same as that in the case of Fig. 
3. In terms of Structure 1 with a relative larger aspect ratio ( $s=2.0$, larger than 0.5 in Fig. 3 ), the displacement mitigation effect of the SDIS is improved in consideration of the coupled SSSI effect, compared to that with only the SSI effect, when the soft soil is considered. Inspired by the soil model expressed in Eq. (4), the reduced structural displacement response benefits from the fact that the SSSI effect enhances the rotational soil spring $k_{s 1}$ (compared to that in the SSI state) to resist the rotation of the foundation. Especially, the stiffness enhancement effect is large in the soft soil condition, and the effect of rotation suppression is transferred and amplified with the height of the structure. Due to the presence of a much taller Structure 2, the relative response between the two buildings can be amplified due to the height difference, which leads to a sufficient utilization of the SDIS for energy absorption and dissipation.

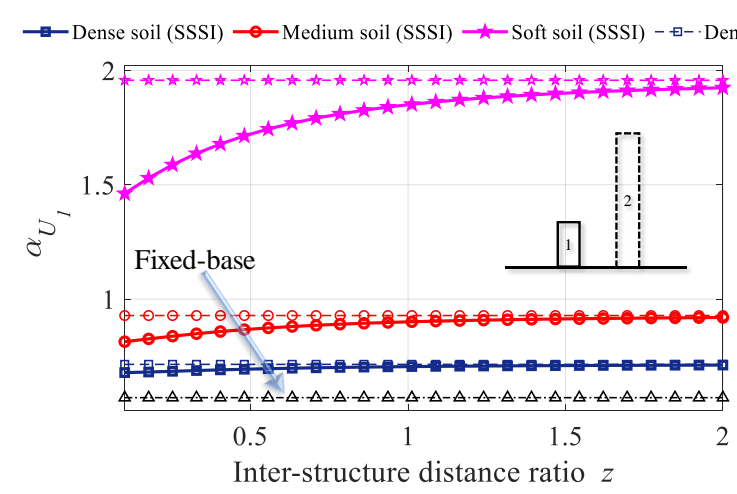

(a)

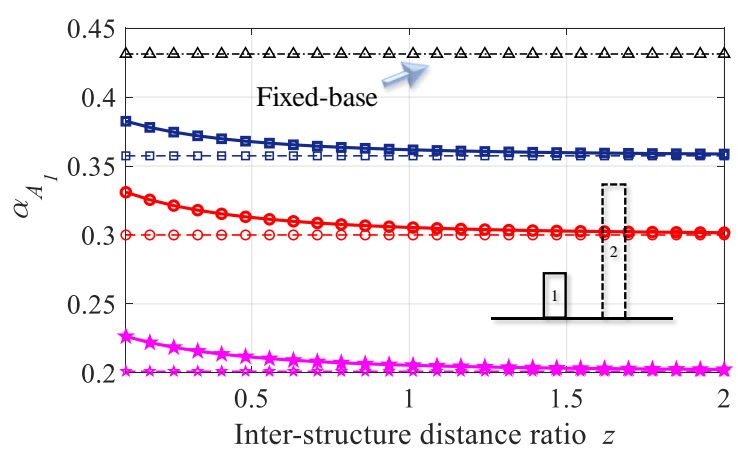

(c)

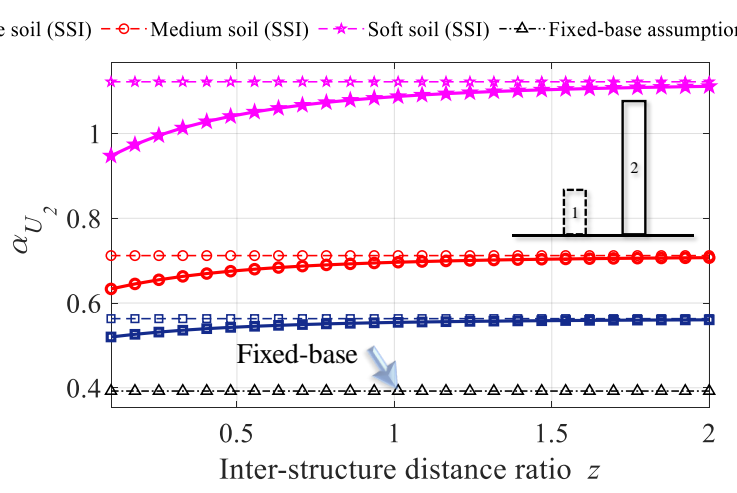

(b)

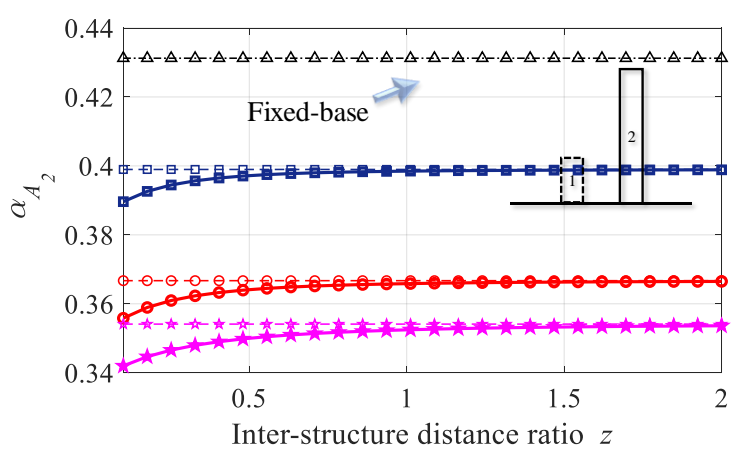

(d)

Fig. 4. Seismic response of AS-SDIS varied with the inter-structure distance ratio $z$ in the case of $(s=2, \varepsilon=3):(\mathrm{a})$ displacement response mitigation ratio of Structure $1 \quad \alpha_{U_{1}} ;(\mathrm{b})$ displacement response mitigation ratio of Structure 2 $\alpha_{U_{2}} ;$ (c) acceleration response mitigation ratio of Structure $1 \alpha_{A_{1}} ;(\mathrm{d})$ acceleration response mitigation ratio of Structure $2 \alpha_{A_{2}}$.

\subsubsection{Influence of height ratio $\varepsilon$ and aspect ratio $s$}

This section aims to explore how the seismic responses of the AS-SDIS vary with the shape of the two structures, i.e., the height ratio $\varepsilon$ and aspect ratio $s$ considered in this study for a given inter-structure distance ratio $z=0.10$ (to represent a pair of closely spaced structures) and the SDIS used in Section 3.2.1. The analysis results of $\alpha_{U_{i}}(\varepsilon, s)$ and $\alpha_{A_{i}}(\varepsilon, s)$ are plotted in Fig. 5-Fig. 7, where the SSI state is represented by the surface with yellow dots and the SSSI state 
is shown in the transparent surface marked by black lines.

Because of the soil effect mentioned in the previous section, the displacement ratio of $\alpha_{U_{i}}$ with the SSI or SSSI effect to $\alpha_{U_{i} \text {, Fixed-base }}$ is larger than one, whereas the acceleration ratio of $\alpha_{A_{i}}$ with the SSI or SSSI effects to $\alpha_{A_{i} \text {, Fixed-base }}$ is lower. It should be noted that the increasing trend of displacement and the decreasing trend of acceleration become more significant as the soil beneath the structures becomes more flexible, ignoring the values of height ratio $\varepsilon$ and aspect ratio $s$. In this situation, the fixed-base assumption without the consideration of soil effects is inaccurate to evaluate the seismic performance of the AS-SDIS. The mentioned SSI or SSSI effects need to be incorporated into the analysis of the AS-SDIS and the design of SDIS parameters.

Comparing the results in the coupled and uncoupled SSI states, it can be observed that the height ratio $\varepsilon$ plays a more important role to determine the seismic responses of the AS-SDIS than the height ratio $s$ of Structure 1 . When Structure 1 is spaced closely to a taller Structure $2(\varepsilon>1)$, the displacement (Fig. 5) and acceleration (Fig. 7) responses of Structure 1 are increased more in the case of the SSSI state than that of the SSI state. This increasing extent is relatively large, especially in the soft soil condition. If the aspect ratio of Structure 1 is very large, i.e., it is a slender structure, its displacement response is suppressed more by the SSSI effect than by the SSI effect, and the substantial reason has been explained in Fig. 4. In terms of Structure 2 in this situation $(\varepsilon>1)$, the lower Structure 1 can behave like a mass damper tuned to Structure 2 and connected by the inter-structure spring $k_{\theta}$, correspondingly reducing the seismic response (Fig. 6 and Fig. 8) of Structure 2 compared with that influenced by the SSI effect. Note that, considering the symmetry characteristic of the studied AS-SDIS, the analysis results and principle of Structure 1 in the case of $\varepsilon>1$ hold true for Structure 2 in the case of $\varepsilon<1$.

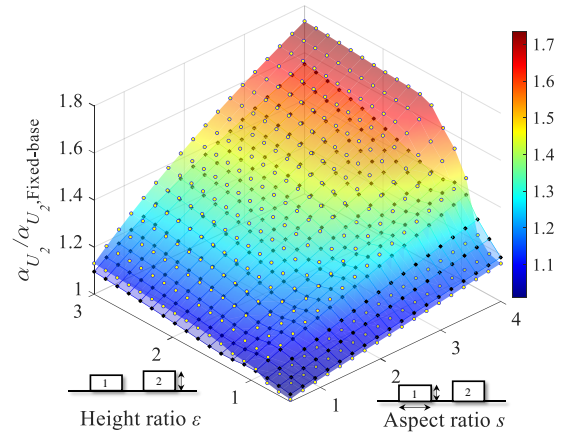

(a)

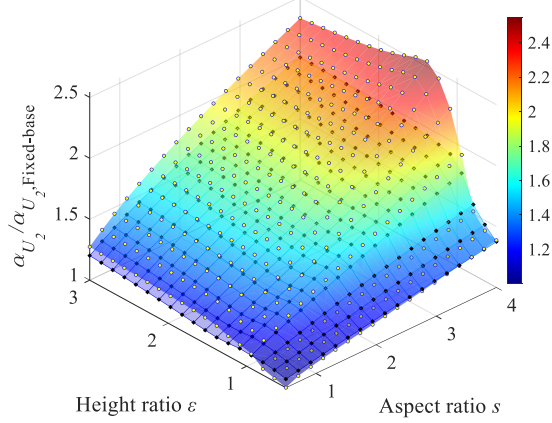

(b)

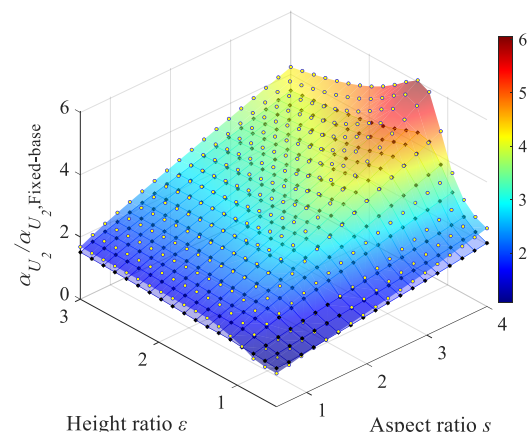

(c)

Fig. 5. Results of displacement response mitigation ratio of Structure $1 \alpha_{U_{1}}$ influenced by SSI effect (surface with yellow dots) and SSSI effect (transparent surface marked by black lines) in the case of $z=0.1$ with varying aspect ratio $s$ and height ratio $\varepsilon:$ (a) dense soil; (b) medium soil; (c) soft soil. 


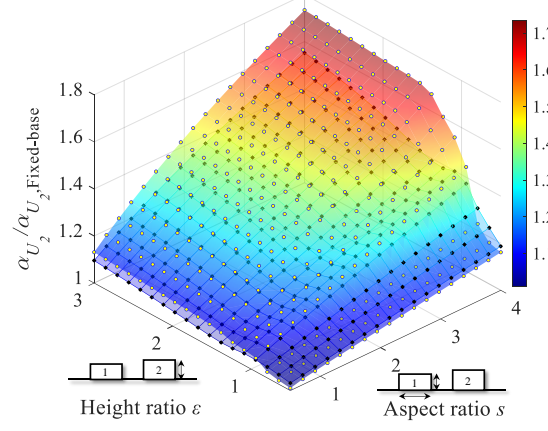

(a)

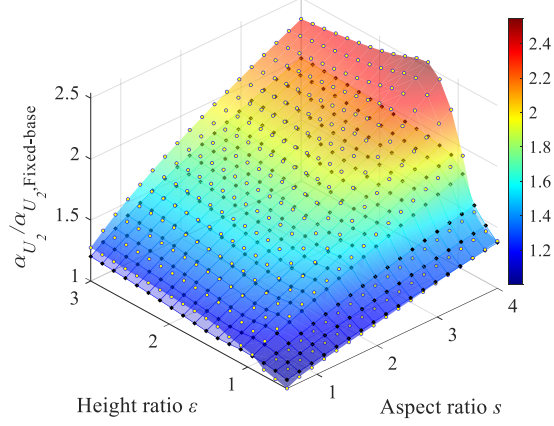

(b)

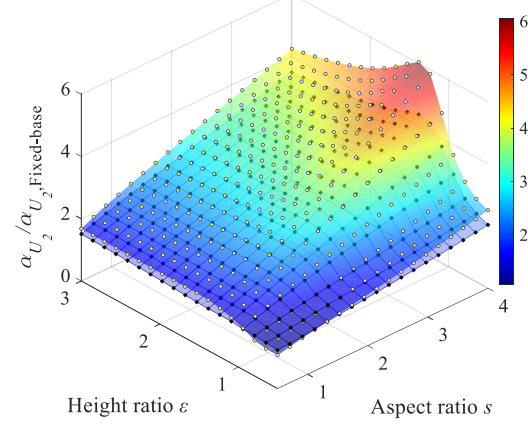

(c)

Fig. 6. Results of displacement response mitigation ratio of Structure $2 \alpha_{U_{2}}$ influenced by the SSI effect (surface with yellow dots) and SSSI effect (transparent surface marked by black lines) in the case of $z=0.1$ with varying aspect ratio $s \in[0.5,4.0]$ and height ratio $\varepsilon \in[0.5,3.0]$ : (a) dense soil; (b) medium soil; (c) soft soil.

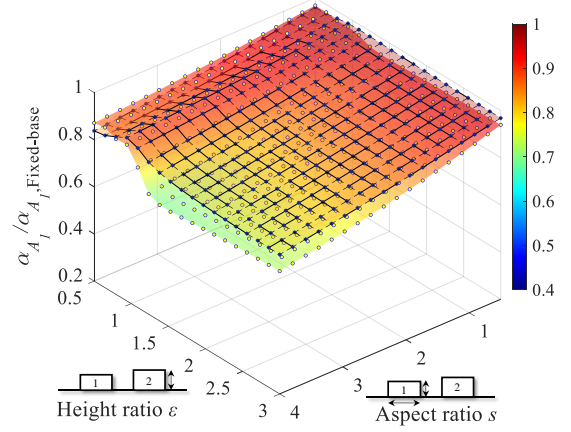

(a)

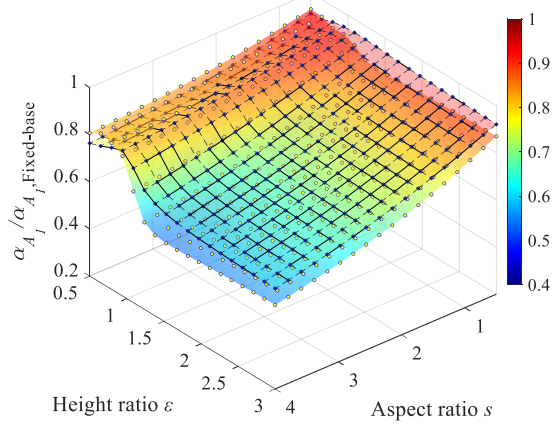

(b)

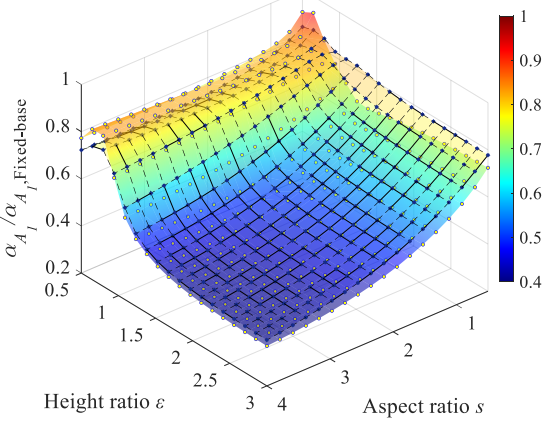

(c)

Fig. 7. Results of acceleration response mitigation ratio of Structure $1 \alpha_{A_{1}}$ influenced by the SSI effect (surface with yellow dots) and SSSI effect (transparent surface marked by black lines) in the case of $z=0.1$ with varying aspect ratio $s \in[0.5,4.0]$ and height ratio $\varepsilon \in[0.5,3.0]$ : (a) dense soil; (b) medium soil; (c) soft soil.

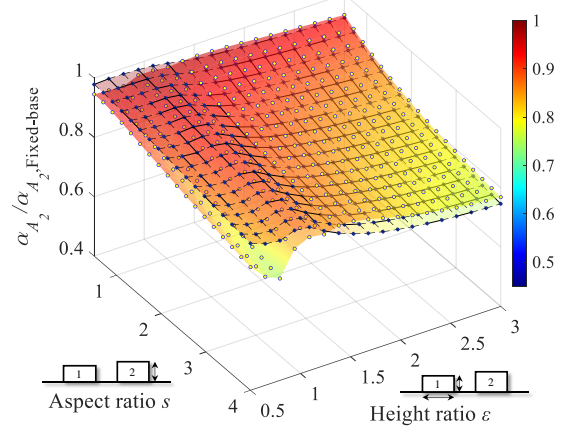

(a)

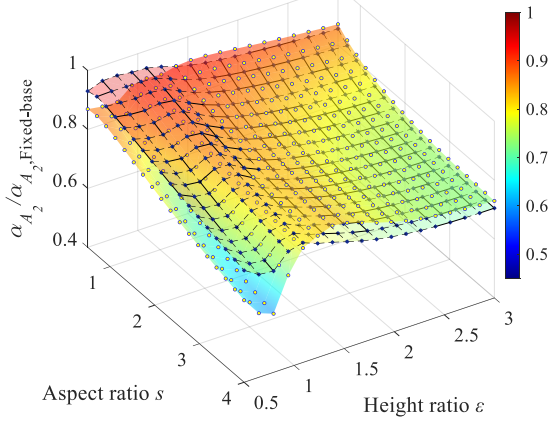

(b)

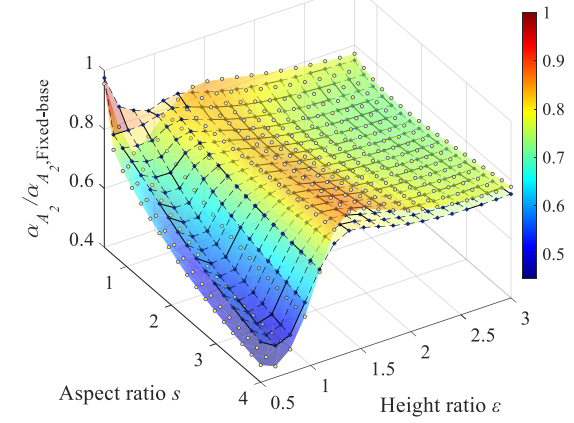

(c)

Fig. 8. Results of acceleration response mitigation ratio of Structure $2 \alpha_{A_{2}}$ influenced by the SSI effect (surface with yellow dots) and SSSI effect (transparent surface marked by black lines) in the case of $z=0.1$ with varying aspect ratio $s \in[0.5,4.0]$ and height ratio $\varepsilon \in[0.5,3.0]$ : (a) dense soil; (b) medium soil; (c) soft soil. 


\subsubsection{Influence of SDIS parameters}

As discussed in the previous section, the SSI and SSSI effects substantially increase the seismic responses, especially the displacement of the lower of two adjacent structures, ignoring the inter-structure distance, structural aspect ratio, and relative height difference between the two adjacent structures. To extensively investigate the soil effect in the case of a SDIS with various parameter sets, i.e., $\mu, \kappa$, and $\xi$, this section reports the analysis results of $\alpha_{U_{i}}(\mu, \kappa, \xi)$ and $\alpha_{A_{i}}(\mu, \kappa, \xi)$ for a given inter-structure distance ratio $z=0.10$ and the adjacent structures used in Section 3.2.1. Additionally, a control force ratio $\alpha_{F}$ is defined in this section to evaluate the control cost and control effect simultaneously, whose detailed expression is,

$$
\alpha_{F, \text { SSSI }}=\frac{[\mathrm{E}(\text { Force of SDIS })]_{\text {SSSI }}}{[\mathrm{E}(\text { Shear force of Strucutre } 1)]_{\text {Uncontrolled }}}, \alpha_{F, \text { SSI }}=\frac{[\mathrm{E}(\text { Force of SDIS })]_{\text {SSI }}}{[\mathrm{E}(\text { Shear force of Strucutre } 1)]_{\text {Uncontrolled }}} .
$$

Fig. 9-Fig. 11 showed the surf plots of $\alpha_{U_{1}}, \alpha_{U_{2}}$, and $\alpha_{F}$ in a medium soil condition as an example. The conclusions of the analysis obtained from this example also hold true for the other adjacent structures with different $s$ and $\varepsilon$. Linked by the SDIS, the two adjacent structures exhibit larger displacement responses and larger control force compared with the AS-SDIS assumed on a fixed base, ignoring the values of SDIS parameters. In this situation, the acceleration responses are lower, as analyzed in the previous section; the results are not reported here owing to the limited space.

Comparing the results of $\alpha_{U_{1}}$ in the SSI and SSSI effects (Fig. 9), it can be concluded that the coupled SSSI effect further amplifies the displacement response of Structure 1, in comparison to the uncoupled SSI effect, when the two adjacent structures are linked by an SDIS with a soft spring and a dashpot with small damping ratio. This soft connection exhibits a weak vibration mitigation effect and is not sufficient to suppress the seismic response of the adjacent structures when the negative influence of the inter-structure spring $k_{\theta}$ is applied owing to the SSSI effect. The increasing trend caused by small $\kappa$ and $\xi$ holds true for the AS-SDIS with different aspect ratios $s$ and height ratios $\varepsilon$. In the situation of Structure 1 with a taller Structure 2, its displacement response is amplified owing to the presence of the taller Structure 2. On the contrary, in most cases of the SDIS with different parameter sets, the displacement responses of Structure 2 (Fig. 10) are substantially reduced by the SSSI effect compared with the SSI effect, owing to the tuning-like effect of Structure 1. With regard to the control force of the SDIS, the value in the SSSI state is slightly larger than that in the SSI state, and the variation trend differs from that of $\alpha_{U_{1}}$ and $\alpha_{U_{2}}$. 


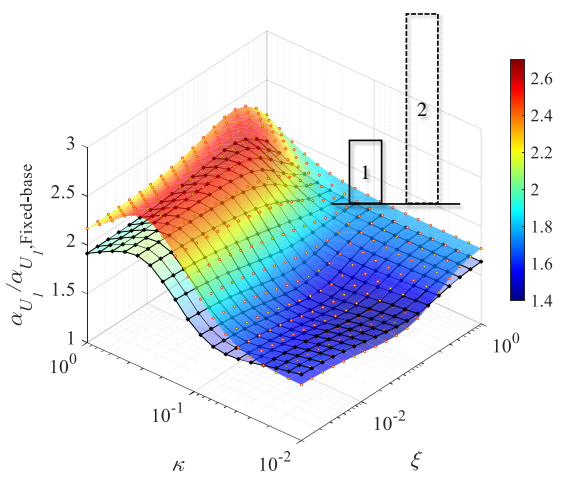

(a)

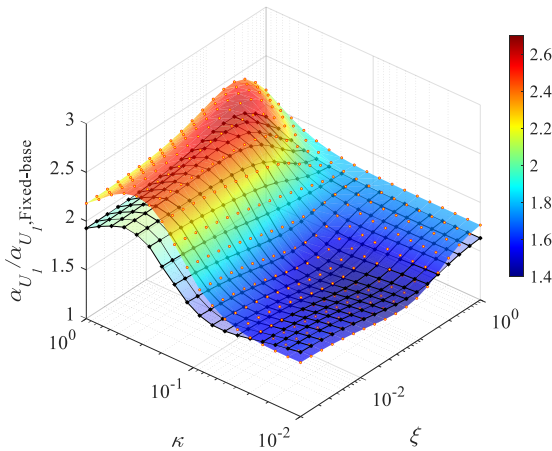

(b)

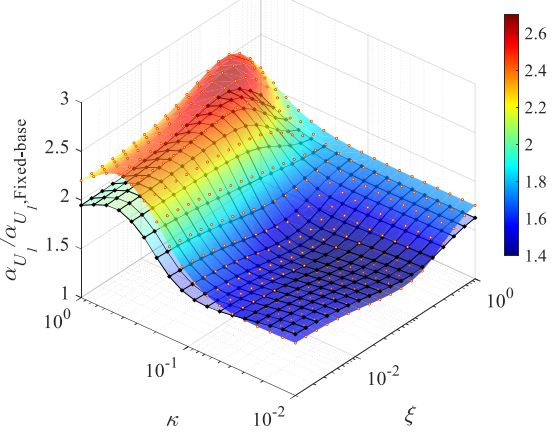

(c)

Fig. 9. Results of displacement response mitigation ratio of Structure $1 \alpha_{U_{1}}$ influenced by the SSI effect (surface with yellow dots) and SSSI effect (transparent surface marked by black lines) in the case of $z=0.1, s=2.0$, and $\varepsilon=3.0$ with varying stiffness ratio $\kappa$ and damping ratio $\xi$ : (a) $\mu=0.1$; (b) $\mu=0.25$; (c) $\mu=0.5$.

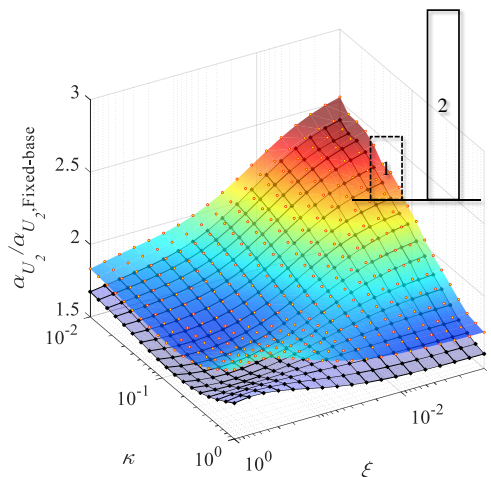

(a)

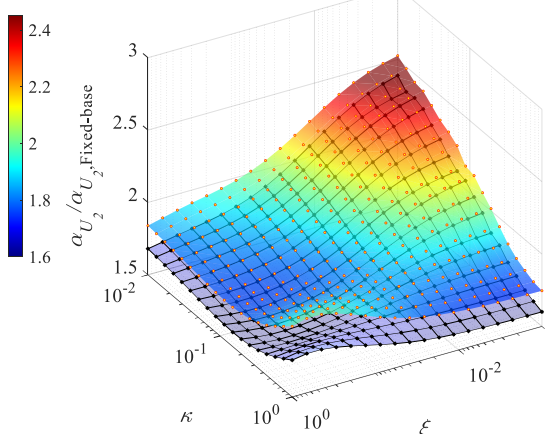

(b)
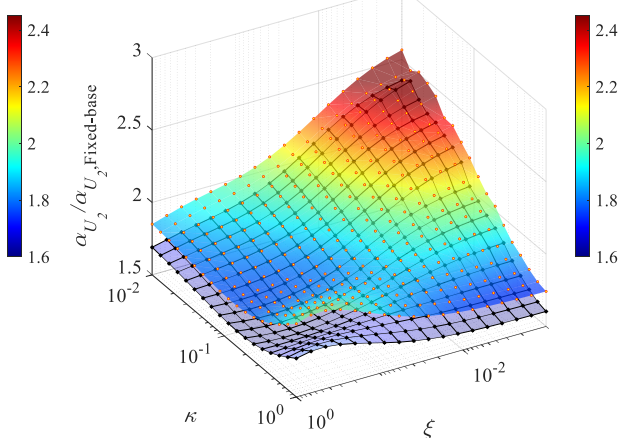

(c)

Fig. 10. Results of displacement response mitigation ratio of Structure $2 \alpha_{U_{2}}$ influenced by SSI effect (surface with yellow dots) and SSSI effect (transparent surface marked by black lines) in the case of $z=0.1, s=2.0$, and $\varepsilon=3.0$ :

(a) $\mu=0.1$; (b) $\mu=0.25$; (c) $\mu=0.5$.

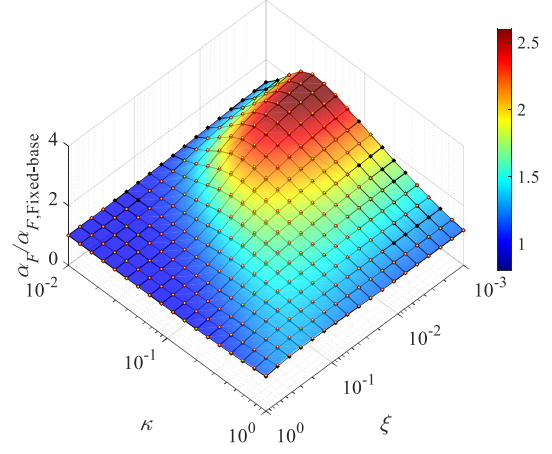

(a)

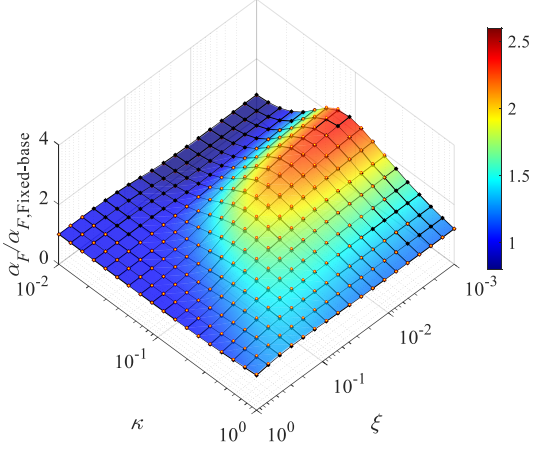

(b)

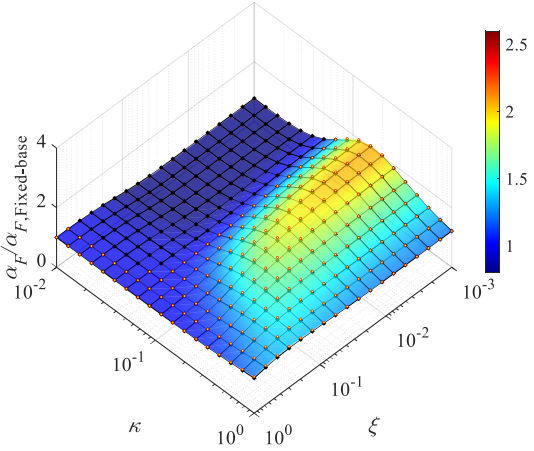

(c)

Fig. 11. Results of control force ratio $\alpha_{F}$ influenced by SSI effect (surface with yellow dots) and SSSI effect (transparent surface marked by black lines) in the case of $z=0.1, s=2.0$, and $\varepsilon=3.0:$ (a) $\mu=0.1$; (b) $\mu=0.25$; (c) $\mu=0.5$. 


\subsection{Time history analysis}

Additionally, for a more extensive exploration of the seismic performance of the AS-SDIS influenced by SSI or coupled SSSI effects, we conduct an analysis in the time domain. Except for the displacement and acceleration responses of the two adjacent structures, the deformation of the SDIS dashpot and energy dissipation effect are also indices of interest, and detailed and intuitive measurements are carried out to illustrate the SSI and coupled SSSI effects.

Linking the adjacent structures by an SDIS, the soil conditions of the structures were considered in the fixed-base, SSI, and SSSI states, and 10 natural records were used, chosen from the Imperial Valley (1940), Kern County (1952), Morgan Hill (1984), Kobe (1995), Loma Prieta (1989), Northridge (1994), Kocaeli (1999), Chi-Chi (1999), Niigata Japan (2004), and Iwate Japan (2008) earthquakes. The corresponding data were obtained from the ground motion database of the Pacific Earthquake Engineering Research Centre (PEER) [49]. The acceleration spectrums for these earthquakes with 5\% damping ratio are plotted in Fig. 12, and their unique record sequence number (RSN) in PEER is given in the legend. The frequency content of these ground motions covers a frequency range from $0.20 \mathrm{~s}$ to $2.00 \mathrm{~s}$.

As an example, the adjacent structures $(s=0.5, \varepsilon=1.25)$ used in Section 3.2.1 linked by an SDIS ( $\mu=0.30, \kappa=0.10$, $\xi=0.05$ ), comprising a soft spring and a dashpot with a small damping ratio, are analyzed. We examine the case when the two structures are closely spaced, with a given inter-distance ratio 0.10 , in the soft soil condition. Table 3 and

Table 4 summarize the response results $\alpha_{U_{i}}$ and $\alpha_{A_{i}}$ of the two structures for the given ground motions. The natural periods of the involved AS-SDIS are $0.53 \mathrm{~s}$ and $0.66 \mathrm{~s}$, which fall in the frequency content of the ground motions. The data marked by an underline indicates that the ratio is greater than 1, that is to say, under the influence of SSI (or SSSI), the structural responses are amplified compared to the assumption of the fixed-base. This increase holds true in most cases of the considered ground motions, substantially requiring the consideration of the interaction between structure and soil for the analysis of AS-SDIS built on the soft soil.

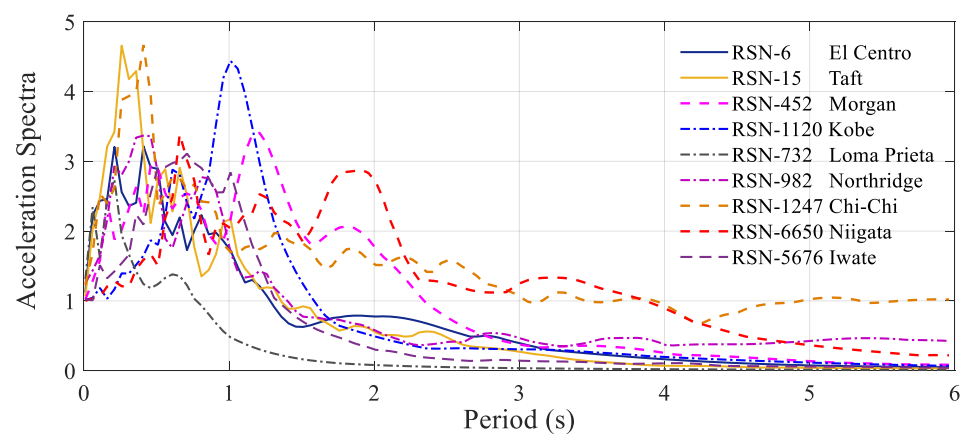

Fig. 12. Normalized acceleration spectra of the recorded earthquakes.

Table 3. Analysis results of seismic responses of Structure 1 in the case of selected ground motions.

\begin{tabular}{llllll}
\hline Results & El Centro & Taft & Morgan & Kobe & Loma Prieta \\
\hline
\end{tabular}




\begin{tabular}{|c|c|c|c|c|c|c|}
\hline$\alpha_{U_{1}, \text { Fixed-base }}$ & & 0.69 & 0.94 & 1.43 & 0.98 & 1.15 \\
\hline$\alpha_{U_{1}} / \alpha_{U_{1}, \text { Fixed-base }}$ & $\begin{array}{l}\text { SSI } \\
\text { SSSI }\end{array}$ & $\frac{1.05}{1.38}$ & $\frac{1.22}{1.41}$ & $\frac{1.46}{1.90}$ & $\underline{1.03}$ & $\frac{2.47}{2.90}$ \\
\hline$\alpha_{A_{1}, \text { Fixed-base }}$ & & 0.60 & 0.82 & 1.25 & 0.89 & 1.02 \\
\hline$\alpha_{A_{1}} / \alpha_{A_{1}, \text { Fixed-base }}$ & $\begin{array}{l}\text { SSI } \\
\text { SSSI }\end{array}$ & $\begin{array}{l}0.57 \\
0.73\end{array}$ & $\begin{array}{l}0.66 \\
0.74\end{array}$ & $\begin{array}{l}0.79 \\
1.01 \\
\end{array}$ & $\begin{array}{l}0.56 \\
0.60\end{array}$ & $\frac{1.33}{1.56}$ \\
\hline Results & & Northridge & Kocaeli & Chi-Chi & Niigata & Iwate \\
\hline$\alpha_{U_{1}, \text { Fixed-base }}$ & & 1.47 & 0.65 & 0.86 & 1.29 & 1.43 \\
\hline$\alpha_{U_{1}} / \alpha_{U_{1}, \text { Fixed-base }}$ & $\begin{array}{l}\text { SSI } \\
\text { SSSI }\end{array}$ & $\underline{1.97}$ & $\underline{1.52}$ & $\underline{1.58}$ & $\underline{2.98}$ & $\underline{1.64}$ \\
\hline$\alpha_{A_{1}, \text { Fixed-base }}$ & & 1.26 & 0.58 & 0.77 & 1.14 & 1.25 \\
\hline$\alpha_{A_{1}} / \alpha_{A_{1}, \text { Fixed-base }}$ & $\begin{array}{l}\text { SSI } \\
\text { SSSI }\end{array}$ & $\frac{1.11}{1.33}$ & $\begin{array}{l}0.80 \\
0.79\end{array}$ & $\begin{array}{l}0.85 \\
0.86\end{array}$ & $\frac{1.61}{1.96}$ & $\begin{array}{l}0.87 \\
0.79\end{array}$ \\
\hline
\end{tabular}

Table 4. Analysis results of seismic responses of Structure 2 in the case of selected ground motions.

\begin{tabular}{|c|c|c|c|c|c|c|}
\hline \multirow{3}{*}{$\begin{array}{l}\text { Results } \\
\alpha_{U_{2}, \text { Fixed-base }} \\
\alpha_{U_{2}} / \alpha_{U_{2}, \text { Fixed-base }}\end{array}$} & & El Centro & Taft & Morgan & Kobe & Loma Prieta \\
\hline & & 0.34 & 0.54 & 0.60 & 0.71 & 0.51 \\
\hline & $\begin{array}{l}\text { SSI } \\
\text { SSSI }\end{array}$ & $\frac{1.36}{1.15}$ & $\frac{1.61}{1.24}$ & $\underline{1.42}$ & $\underline{1.79}$ & $\underline{2.20}$ \\
\hline \multirow{2}{*}{$\begin{array}{l}\alpha_{A_{2}, \text { Fixed-base }} \\
\alpha_{A_{2}} / \alpha_{A_{2} \text {,Fixed-base }}\end{array}$} & & 0.37 & 0.59 & 0.65 & 1.11 & 0.51 \\
\hline & $\begin{array}{l}\text { SSI } \\
\text { SSSI } \\
\end{array}$ & $\begin{array}{l}0.71 \\
0.75 \\
\end{array}$ & $\begin{array}{l}0.86 \\
0.80 \\
\end{array}$ & $\begin{array}{l}0.74 \\
0.90 \\
\end{array}$ & $\begin{array}{l}0.92 \\
0.86 \\
\end{array}$ & $\begin{array}{l}1.22 \\
1.18 \\
\end{array}$ \\
\hline Results & & Northridge & Kocaeli & Chi-Chi & Niigata & Iwate \\
\hline \multirow{2}{*}{$\begin{array}{l}\alpha_{U_{2}, \text { Fixed-base }} \\
\alpha_{U_{2}} / \alpha_{U_{2}, \text { Fixed-base }}\end{array}$} & & 0.42 & 0.74 & 0.44 & 0.44 & 0.67 \\
\hline & $\begin{array}{l}\text { SSI } \\
\text { SSSI }\end{array}$ & $\underline{1.27}$ & $\begin{array}{l}2.44 \\
\underline{1.68} \\
\end{array}$ & $\underline{1.78}$ & $\underline{1.74}$ & $\begin{array}{l}\underline{2.38} \\
\underline{1.67} \\
\end{array}$ \\
\hline \multirow{2}{*}{$\begin{array}{l}\alpha_{A_{2}, \text { Fixed-base }} \\
\alpha_{A_{2}} / \alpha_{A_{2}, \text { Fixed-base }}\end{array}$} & & 0.43 & 0.81 & 0.47 & 0.45 & 0.73 \\
\hline & $\begin{array}{l}\text { SSI } \\
\text { SSSI } \\
\end{array}$ & $\begin{array}{l}0.81 \\
0.91 \\
\end{array}$ & $\underline{1.21}$ & $\begin{array}{l}0.94 \\
0.87 \\
\end{array}$ & $\begin{array}{l}0.97 \\
1.12 \\
\end{array}$ & $\frac{1.20}{0.99}$ \\
\hline
\end{tabular}

As an example, Fig. 13 and Fig. 14 show the seismic responses, namely, the defined $U_{1}$ and $A_{1}$ for Structure 1 of the AS-SDIS in the states of fixed-base (red line), uncoupled SSI (dotted line), and SSSI (dark blue line). Comparing the responses of Structure 1 on the fixed base and soft soil including the SSI and SSSI effects, we observe that the maximum displacement and acceleration responses are larger owing to the soil effect. In terms of the comparison between the SSI and SSSI response, Structure 1 appears to undergo a more detrimental interaction effect when the coupling mechanism (SSSI) is considered.

Fig. 15 shows the corresponding transfer function curves of the displacement $U_{i}$ considering the three states, where the displacements of both structures are amplified owing to the soil effects. Comparing the response results of the AS-SDIS in SSI and SSSI states (Fig. 15(a)), the lower Structure 1 is significantly affected by the taller Structure 2, where big amplifications in the displacement and acceleration are found when the frequency ratio is approximately 0.75 (mainly representing the case where the excitation frequency is close to the vibration frequency of Structure 1 influenced by soil). With regard to $U_{2}$, it is exhibited a delivery of the seismic energy from Structure 2 (characterized by the first peak with a frequency ratio of 0.60 ) to Structure 1 (characterized by the second peak with a frequency ratio of 0.75 ) owing to the 
coupling effect.

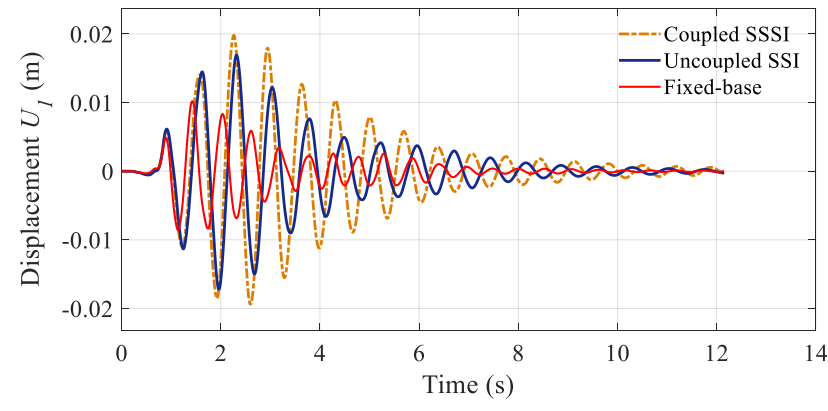

(a) Northridge

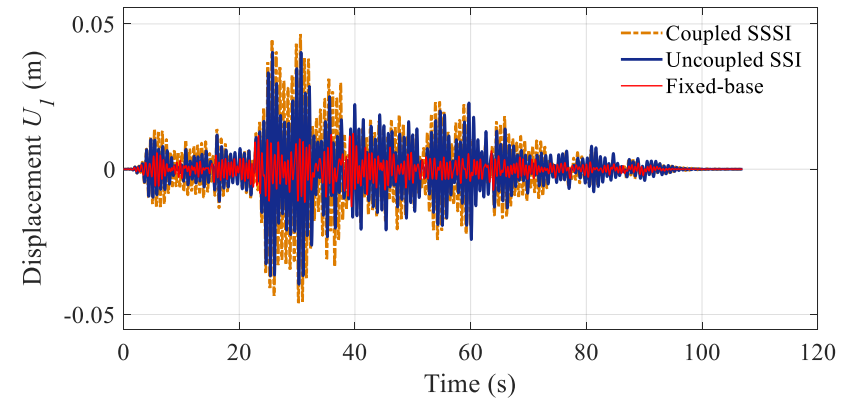

(b) Niigata Japan

Fig. 13. Displacement responses $U_{1}$ of Structure 1 of the AS-SDIS founded on the fixed base and flexible soil.

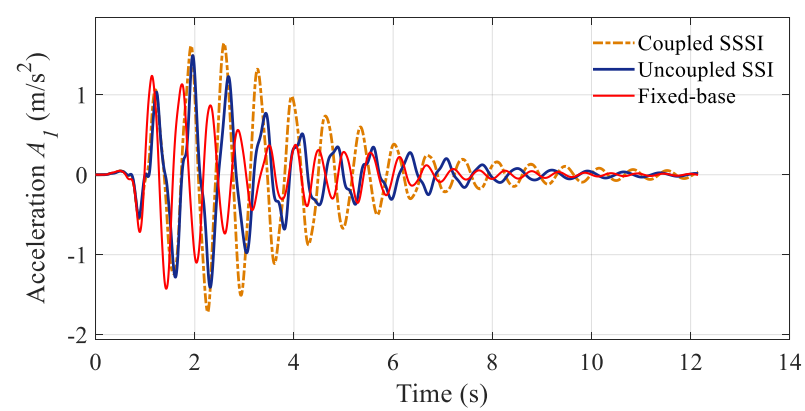

(a) Northridge

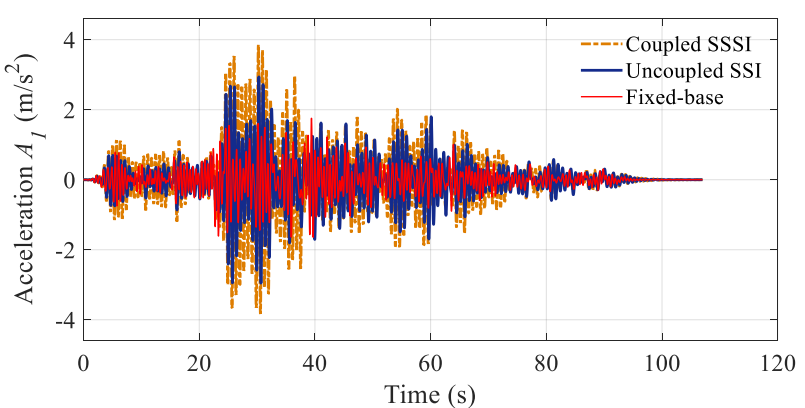

(b) Niigata Japan

Fig. 14. Acceleration responses $A_{1}$ of Structure 1 of the AS-SDIS founded on the fixed base and flexible soil.

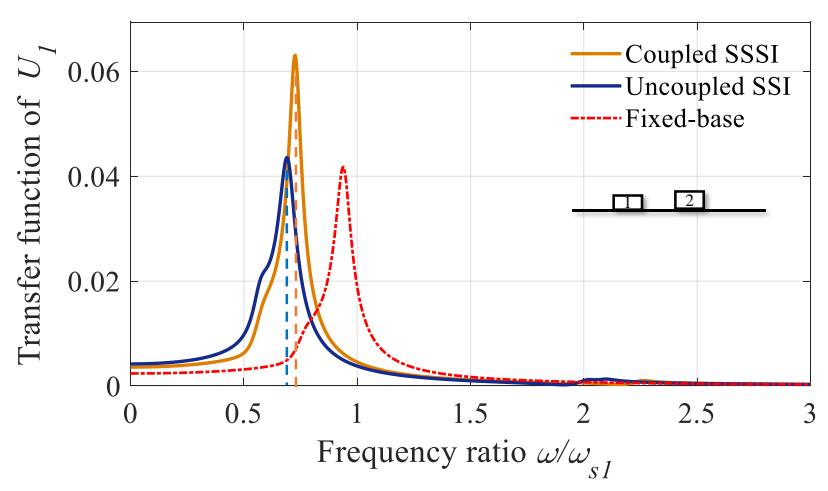

(a) Transfer function of $U_{1}$ for Structure 1

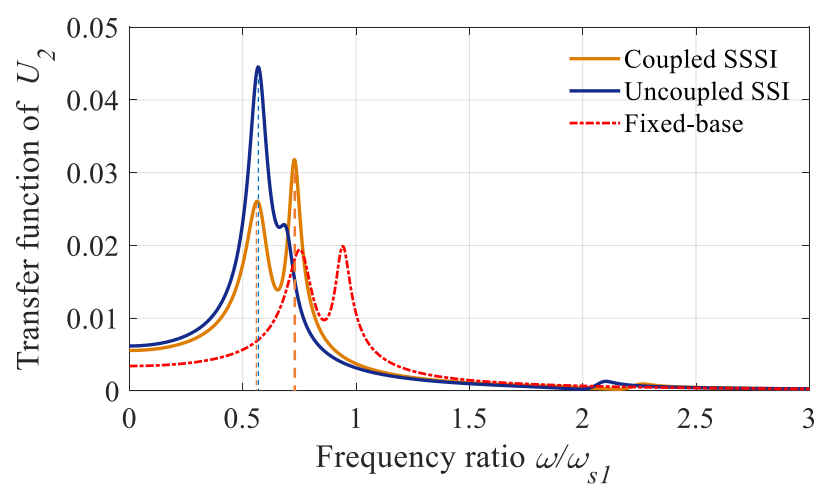

(b) Transfer function of $U_{2}$ for Structure 2

Fig. 15. Transfer functions of AS-SDIS in soft soil condition for a parameter set $(z=0.10, \varepsilon=1.25, s=0.5)$.

From the perspective of an energy-based evaluation, part of the excitation-induced input seismic energy is dissipated by the two primary structures, and the remaining part is dissipated by the SDIS in the case of fixed-base. Incorporating the soil effect into the AS-SDIS, a portion of the energy preliminary dissipated by the primary structures or SDIS is dissipated by the soil as a substitute. To quantify this transfer of seismic energy from the structure into the soil, this section conducted an energy analysis referring to Eq. (2) by pre-multiplication by $\mathbf{u}^{T}$ and integration over the time domain. The balanced 
relationship of the dissipated energy is classified and given by

$$
E_{\text {total }}(t)=E_{S}(t)+E_{S D I S}(t)+E_{\text {Soil }}(t)
$$

where the total dissipated energy $E_{\text {total }}(t)$ is composed of the contributions of the structural inherent damping dissipated energy $E_{S}(t)$ (including Structure 1 and Structure 2), the SDIS-dissipated energy $E_{S D I S}(t)$, and soil-dissipated energy $E_{\text {Soil }}(t)$ (especially is zero in the case of fixed-base condition).

Fig. 16 shows the energy curves for the AS-SDIS in the fixed-base, SSI, and SSSI states, whose parameters are the same as those of the case in Section 4.1. Comparing the maximum total seismic input energy, we observe a remarkable increase when the soil effect is incorporated in the analysis of the AS-SDIS (Fig. 16(b) and (c)) in comparison to the case in Fig. 16(a). Correspondingly, more energy is dissipated by the SDIS and soil. Compared with the results of the fixed-base case shown in Fig. 17, the soil effect (SSI and SSSI) introduces the rotation DOF into the AS-SDIS and substantially amplifies the relative displacement of the two adjacent structures (which is equal to the SDIS deformation). The increased relative displacement response potentially increases the risk of accidental pounding in adjacent structures and causes the SDIS to deform beyond the allowable value of the device.

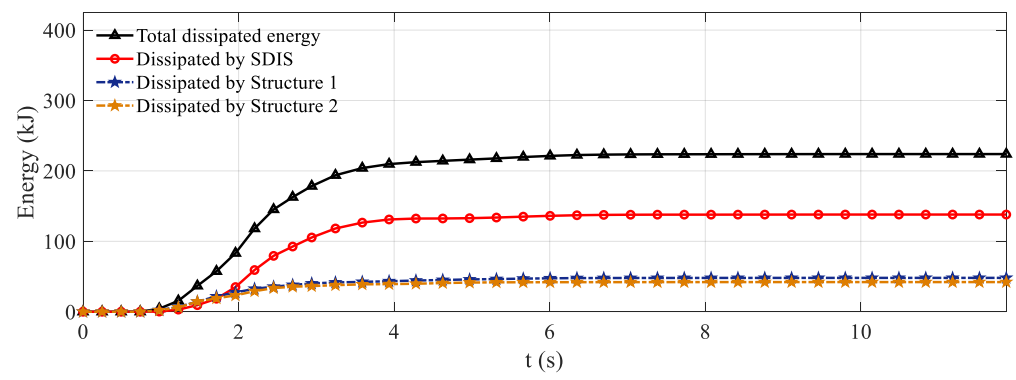

(a) Structure on fixed base

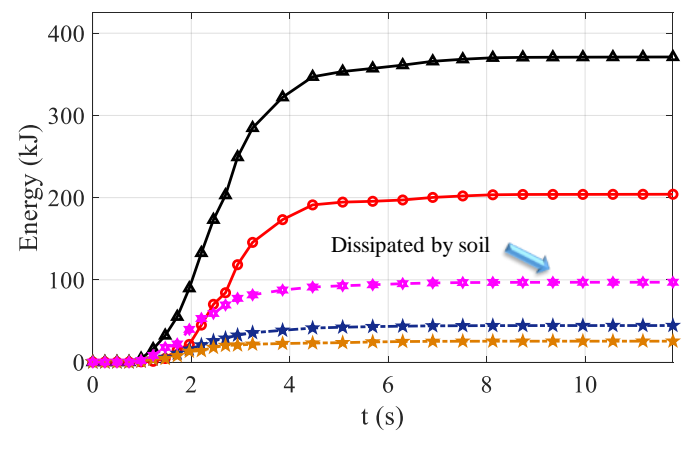

(b) Structure in SSI state

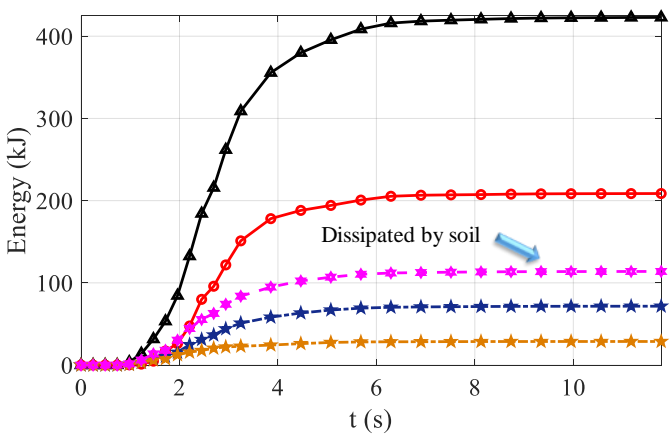

(c) Structure in SSSI state

Fig. 16. Energy curves of AS-SDIS founded on the fixed base and flexible soil under the excitation of Northridge earthquake. 


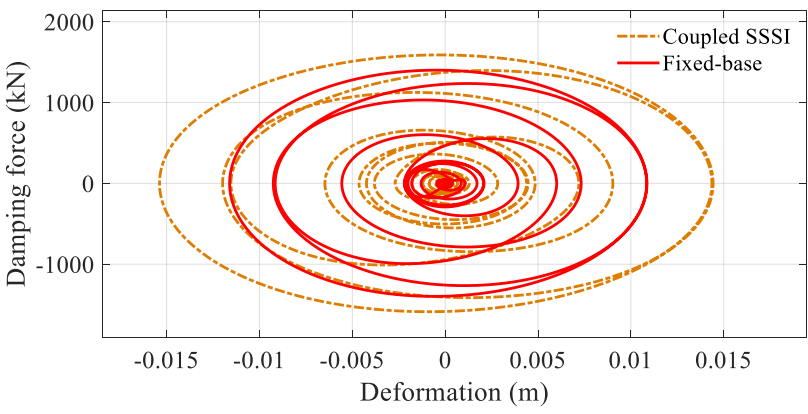

(a) SSSI state

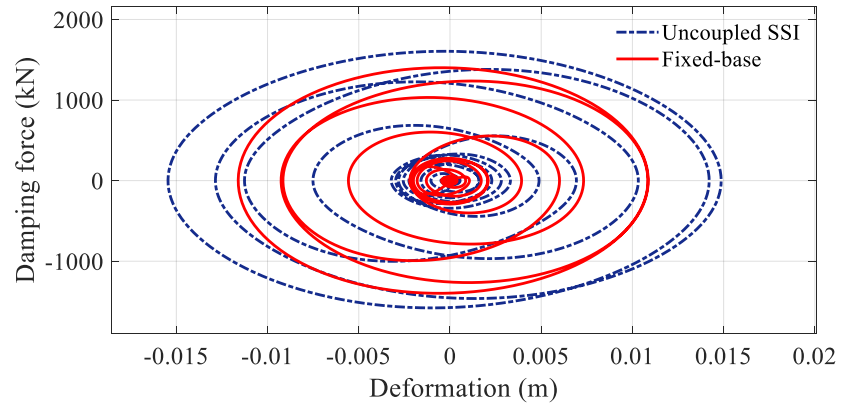

(b) SSI state

Fig. 17. Hysteretic curves of the dashpot in the SDIS for structures founded on fixed-base and flexible soil under the excitation of the Northridge earthquake.

\section{Optimal design framework for AS-SDIS considering soil condition}

\subsection{Design methodology}

The current design method of adjacent structures linked by an inerter-based system is restricted by the assumption of a fixed base, without taking into account the soil condition. Considering the interaction between the soil and AS-SDIS, it can be concluded from the extensive parametric analysis that the SDIS demonstrates a reduced vibration control effect of the two adjacent structures. Therefore, it is suggested that the soil effect be properly considered in the process of SDIS design. The pursued aim is to minimize at the same time the displacement responses of the two adjacent structures, i.e., $\alpha_{U_{1}}$ and $\alpha_{U_{2}}$, which represents a dual-objective design problem. Formulated based on the parametric analysis in Section 3.2, the variation trend of the displacement control effect $\left(\alpha_{U_{1}}\right.$ and $\left.\alpha_{U_{2}}\right)$ and the control cost evaluated by $\alpha_{F}$ against the SDIS parameters and locations of the two indicators differ from each other. The optimal design of the SDIS with consideration of the soil effect is essentially a trade-off between the displacement performances of the two structures and the control force. Following the performance-demand-based design philosophy, the optimal design of the SDIS affected by the soil is, therefore, formulated as

$$
\left\{\begin{array}{l}
\operatorname{minimize} \quad \alpha_{F}(\mu, \kappa, \xi) \\
\text { subject to } \quad \alpha_{U_{i}}(\mu, \kappa, \xi) \leq \alpha_{U_{i}, \lim }
\end{array},\right.
$$

where $\alpha_{U_{i} \text {,lim }}$ denotes the target displacement response ratio of Structure $i$. Under the premise of $\alpha_{U_{i} \text {,lim }}$, the control force of the SDIS is minimized in the proposed design method to pursue an economical solution.

\subsection{Illustrative design cases}

Utilizing the proposed design method, some examples of typical adjacent structures with SDIS are analyzed in this section with consideration of the soil effect. Supposing a pair of adjacent structures with $s=0.5, \varepsilon=2.0$, and $z=0.1$ 
and founded on a medium soil, the target displacement performances $\alpha_{U_{1}, \text { lim }}$ and $\alpha_{U_{1} \text {,lim }}$ are pre-specified as 0.50 (CaseI), 0.60 (Case-II), and 0.70 (Case-III) as examples, which correspond, respectively, to reductions of 50\%, 40\%, and 30\% of the relative (sway + rotational) displacement of the AS-SDIS-soil system compared to the fixed-base uncontrolled structures. In light of the determined target performance in each case, the SDIS parameters can be obtained numerically from Eqs. (16) and are summarized in Table 5. By inspection of the optimal results, it is noted that a large $\xi$ (corresponding to a dashpot with large damping coefficient) is beneficial for the reduction of structural displacements. At the same time, a large $\mu$ linked between the two structures is effective to reduce the control force of the SDIS. The displacement responses of Cases-I to III are shown in Fig. 18, where the designed SDISs are verified as effective to satisfy the pre-specified target demands for both structures considering the soil effects.

Table 5. Designed SDIS parameters and structural response results.

\begin{tabular}{cccccc}
\hline \multirow{2}{*}{ Case ID } & Pre-specified & \multicolumn{2}{c}{ Optimal SDIS parameters } & $\xi$ & $\alpha_{F}$ \\
& $\alpha_{U_{1}, t}=\alpha_{U_{2}, t}$ & $\mu$ & $\kappa$ & 0.101 & 0.177 \\
Case-I & 0.50 & 0.032 & 0.091 & 0.090 & 0.148 \\
Case-II & 0.60 & 0.129 & 0.077 & 0.056 & 0.136 \\
Case-III & 0.70 & 0.201 & 0.096 & \\
\hline
\end{tabular}

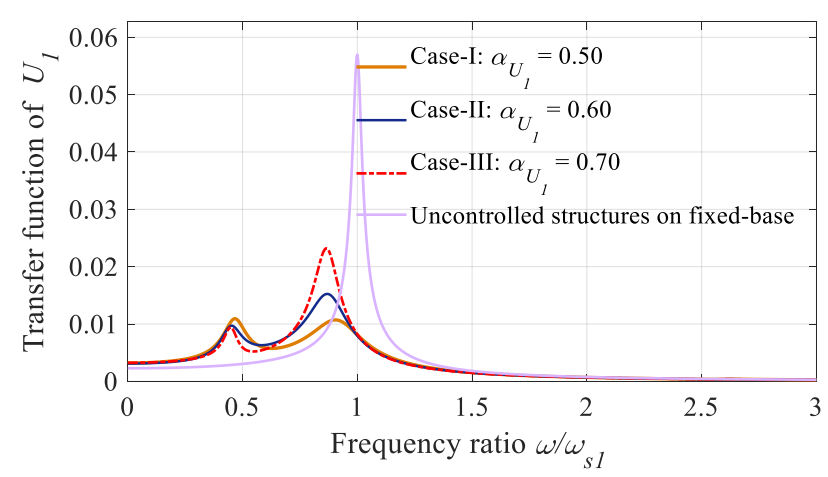

(a) Transfer function of $U_{1}$ for Structure 1

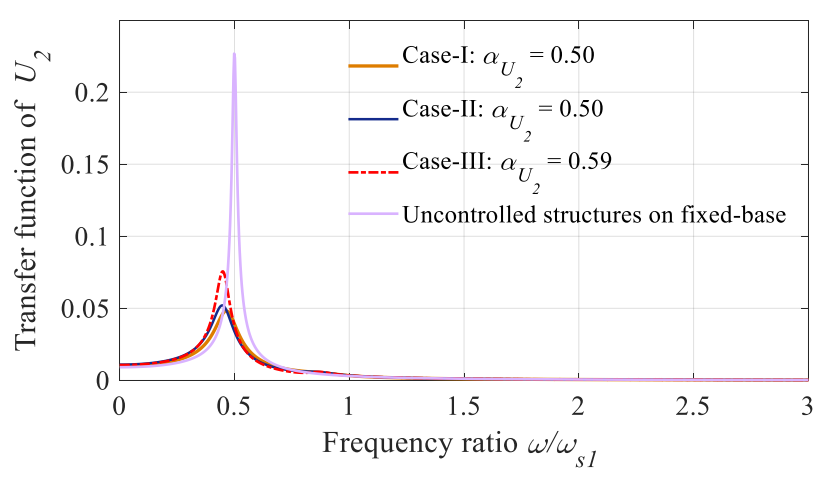

(b) Transfer function of $U_{2}$ for Structure 2

Fig. 18. Transfer functions of adjacent structures with SDIS designed considering soil effects.

\section{Conclusions}

This study dealt with the interaction behavior between adjacent structures interconnected by an inerter system (SDIS) and the underlying soil medium. Considering the soil effects, the dynamic characteristics of the AS-SDIS were revealed in terms of the estimation of the AS-SDIS's dynamic performances and the control mechanism of the interconnected SDIS. A demand-performance-based design method was developed for the SDIS by incorporating the revealed soil effects. Based on the analysis results, the following conclusions can be drawn:

(1) Affected by the underlying soil medium, the SDIS exhibits a weakened vibration mitigation effect for the two adjacent structures, compared with the expected performance assumed by the fixed base. Owing to the influence of soft soil, the fundamental period of the soil-AS-SDIS system is larger than that in the case of the fixed-base assumption, potentially leading to increased displacement responses of the adjacent structures and a decreasing trend of structural acceleration response. 
(2) The coupled SSSI effect plays a significant role in the case of the closely spaced AS-SDIS $(z<1)$. The displacement and acceleration responses of the lower of two adjacent structures are significantly amplified in comparison to the case of a SSI state, which holds true for various parameter sets of SDIS, soil type, and geometric characteristic of structures. The seismic input energy is substantially increased owing to the interaction between the AS-SDIS and soil, leading to the fact that more energy needs to be dissipated by the SDIS through a larger inner deformation of itself.

(3) The developed optimal design method for the SDIS can satisfy the pre-specified target displacement demands for the adjacent structures considering the soil condition. Simultaneously, the control force of the SDIS is optimized with the aid of the designed inertance, accordingly yielding an economical solution to the soil-condition-based control problem of the AS-SDIS.

(4) The research object of this study comprises inerter-based adjacent structures in the linear state. In future studies, the nonlinear behavior of the interaction between the soil and inerter-based structures should be incorporated in the numerical and experimental analysis, and its effect in the corresponding optimal design should be investigated.

\section{Acknowledgements}

This study was supported by the National Natural Science Foundation of China (grant no. 51978525 and 51778489) and the Basic Research Project of the State Key Laboratory of the Ministry of Science and Technology (grant no. SLDRCE19A-02).

\section{References}

[1] S.D. Bharti, S.M. Dumne, M.K. Shrimali, Seismic response analysis of adjacent buildings connected with MR dampers, Engineering Structures, 32 (2010) 2122-2133. https://doi.org/10.1016/j.engstruct.2010.03.015.

[2] K.S. Park, S.Y. Ok, Hybrid control approach for seismic coupling of two similar adjacent structures, Journal of Sound and Vibration, 349 (2015) 1-17. https://doi.org/10.1016/j.jsv.2015.03.028.

[3] M. Basili, M. De Angelis, G. Fraraccio, Shaking table experimentation on adjacent structures controlled by passive and semi-active MR dampers, Journal of Sound and Vibration, $332 \quad$ (2013) 3113-3133. https://doi.org/10.1016/j.jsv.2012.12.040.

[4] Y.L. Xu, S. Zhan, J.M. Ko, W.S. Zhang, Experimental investigation of adjacent buildings connected by fluid damper, Earthquake Engineering \& Structural Dynamics, 28 (1999) 609-631. https://doi.org/10.1002/(sici)10969845(199906)28:6<609::aid-eqe831>3.0.co;2-s.

[5] B.D. Westermo, The dynamics of interstructural connection to prevent pounding, Earthquake Engineering \& Structural Dynamics, 18 (1989) 687-699. https://doi.org/10.1002/eqe.4290180508.

[6] W.S. Zhang, Y.L. Xu, Dynamic characteristics and seismic response of adjacent buildings linked by discrete dampers, Earthquake Engineering \& Structural Dynamics, 28 (1999) 1163-1185. https://doi.org/10.1002/(sici)10969845(199910)28:10<1163::aid-eqe860>3.0.co;2-0.

[7] Y.Q. Ni, J.M. Ko, Z.G. Ying, Random seismic response analysis of adjacent buildings coupled with non-linear hysteretic dampers, Journal of Sound and Vibration, 246 (2001) 403-417. https://doi.org/10.1006/jsvi.2001.3679.

[8] M. Basili, M. De Angelis, A reduced order model for optimal design of 2-mdof adjacent structures connected by hysteretic dampers, Journal of Sound and Vibration, 306 (2007) 297-317. https://doi.org/10.1016/j.jsv.2007.05.012.

[9] M. Basili, M. De Angelis, D. Pietrosanti, Modal analysis and dynamic response of two adjacent single-degree-offreedom systems linked by spring-dashpot-inerter elements, Engineering Structures, 174 (2018) 736-752. https://doi.org/10.1016/j.engstruct.2018.07.048.

[10] K. Ikago, K. Saito, N. Inoue, Seismic control of single-degree-of-freedom structure using tuned viscous mass damper, Earthquake Engineering \& Structural Dynamics, 41 (2012) 453-474. https://doi.org/10.1002/eqe.1138.

[11] S. Kawamata, Development of a vibration control system of structures by means of mass pumps, in, Institute of Industrial Science, University of Tokyo, Tokyo, Japan, 1973.

[12] R.F. Zhang, Z.P. Zhao, C. Pan, K. Ikago, S.T. Xue, Damping enhancement principle of inerter system, Structural Control and Health Monitoring, (2020) e2523. https://doi.org/10.1002/stc.2523.

[13] Z.P. Zhao, R.F. Zhang, Z. Lu, A particle inerter system for structural seismic response mitigation, Journal of the 
Franklin Institute, (2019). https://doi.org/10.1016/j.jfranklin.2019.02.001.

[14] Z.P. Zhao, R.F. Zhang, Y.Y. Jiang, C. Pan, A tuned liquid inerter system for vibration control, International Journal of Mechanical Sciences, (2019) 105171. https://doi.org/10.1016/j.ijmecsci.2019.105171.

[15] R.F. Zhang, Z.P. Zhao, K. Dai, Seismic response mitigation of a wind turbine tower using a tuned parallel inerter mass system, Engineering Structures, 180 (2019) 29-39. https://doi.org/10.1016/j.engstruct.2018.11.020.

[16] Q.J. Chen, Z.P. Zhao, Y.Y. Xia, C. Pan, H. Luo, R.F. Zhang, Comfort based floor design employing tuned inerter mass system, Journal of Sound and Vibration, 458 (2019) 143-157. https://doi.org/10.1016/j.jsv.2019.06.019.

[17] H. Garrido, O. Curadelli, D. Ambrosini, Improvement of tuned mass damper by using rotational inertia through tuned viscous mass damper, Engineering Structures, 56 (2013) 2149-2153. https://doi.org/10.1016/j.engstruct.2013.08.044.

[18] Z.P. Zhao, R.F. Zhang, Y.Y. Jiang, D. De Domenico, C. Pan, Displacement-dependent damping inerter system for seismic response control, Applied Sciences, 10 (2019) 257.

[19] I.F. Lazar, S.A. Neild, D.J. Wagg, Using an inerter-based device for structural vibration suppression, Earthquake Engineering \& Structural Dynamics, 43 (2014) 1129-1147. https://doi.org/10.1002/eqe.2390.

[20] L. Marian, A. Giaralis, Optimal design of a novel tuned mass-damper-inerter (TMDI) passive vibration control configuration for stochastically support-excited structural systems, Probabilistic Engineering Mechanics, 38 (2014) 156-164. https://doi.org/10.1016/j.probengmech.2014.03.007.

[21] M. Basili, M.D. Angelis, D. Pietrosanti, Dynamic response of a viscously damped two adjacent degree of freedom system linked by inerter subjected to base harmonic excitation, Procedia Engineering, 199 (2017) 1586-1591. https://doi.org/10.1016/j.proeng.2017.09.062.

[22] Y. Hu, M.Z.Q. Chen, Z. Shu, L. Huang, Analysis and optimisation for inerter-based isolators via fixed-point theory and algebraic solution, Journal of Sound and Vibration, 346 (2015) 17-36. https://doi.org/10.1016/j.jsv.2015.02.041.

[23] Y.Y. Jiang, Z.P. Zhao, R.F. Zhang, D. De Domenico, C. Pan, Optimal design based on analytical solution for storage tank with inerter isolation system, Soil Dynamics and Earthquake Engineering, 129 (2020) 105924. https://doi.org/10.1016/j.soildyn.2019.105924.

[24] A.R. Ozuygur, A.N. Gunduz, Optimal control of structures under earthquakes including soil-structure interaction, Journal of Earthquake Engineering, 22 (2018) 1317-1335. https://doi.org/10.1080/13632469.2016.1277567.

[25] E. Ahmadi, On the structural energy distribution and cumulative damage in soil-embedded foundation-structure interaction systems, Engineering Structures, 182 (2019) 487-500. https://doi.org/10.1016/j.engstruct.2018.12.091.

[26] E. Ahmadi, Concurrent effects of inertial and kinematic soil-structure interactions on strength-ductility-period

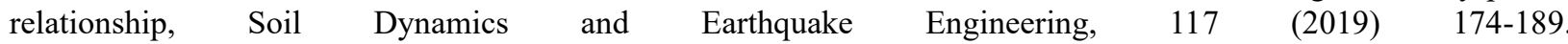
https://doi.org/10.1016/j.soildyn.2018.10.043.

[27] F. Vicencio, N.A. Alexander, A parametric study on the effect of rotational ground motions on building structural responses, Soil Dynamics and Earthquake Engineering, 118 (2019) 191-206. https://doi.org/10.1016/j.soildyn.2018.12.022.

[28] R.N. Jabary, G.S.P. Madabhushi, Tuned mass damper positioning effects on the seismic response of a soil-MDOFstructure system, Journal of Earthquake Engineering, 22 (2018) 281-302. https://doi.org/10.1080/13632469.2016.1224743.

[29] Q.J. Chen, Z.P. Zhao, R.F. Zhang, C. Pan, Impact of soil-structure interaction on structures with inerter system, Journal of Sound and Vibration, 433 (2018) 1-15. https://doi.org/10.1016/j.jsv.2018.07.008.

[30] M. Lou, H. Wang, X. Chen, Y. Zhai, Structure-soil-structure interaction: Literature review, Soil Dynamics and Earthquake Engineering, 31 (2011) 1724-1731. https://doi.org/10.1016/j.soildyn.2011.07.008.

[31] S. Hans, C. Boutin, E. Ibraim, P. Roussillon, In situ experiments and seismic analysis of existing buildings. Part I: experimental investigations, Earthquake Engineering \& Structural Dynamics, 34 (2005) 1513-1529. 10.1002/eqe.502.

[32] R.N. Jabary, S.P.G. Madabhushi, Structure-soil-structure interaction effects on structures retrofitted with tuned mass dampers, Soil Dynamics and Earthquake Engineering, 100 (2017) 301-315. https://doi.org/10.1016/j.soildyn.2017.05.017.

[33] F. Vicencio, N.A. Alexander, Dynamic interaction between adjacent buildings through nonlinear soil during earthquakes, Soil Dynamics and Earthquake Engineering, 108 (2018) 130-141. https://doi.org/10.1016/j.soildyn.2017.11.031.

[34] J.S. Mulliken, D.L. Karabalis, Discrete model for dynamic through-the-soil coupling of 3-D foundations and structures, Earthquake Engineering \& Structural Dynamics, 27 (1998) 687-710. https://doi.org/10.1002/(sici)10969845(199807)27:7<687::aid-eqe752>3.0.co;2-o.

[35] N.A. Alexander, E. Ibraim, H. Aldaikh, A simple discrete model for interaction of adjacent buildings during earthquakes, Computers \& Structures, 124 (2013) 1-10. https://doi.org/10.1016/j.compstruc.2012.11.012.

[36] F. Vicencio, N.A. Alexander, Higher mode seismic structure-soil-structure interaction between adjacent building during earthquakes, Engineering Structures, 174 (2018) 322-337. https://doi.org/10.1016/j.engstruct.2018.07.049.

[37] M. Basili, M. De Angelis, D. Pietrosanti, Defective two adjacent single degree of freedom systems linked by springdashpot-inerter for vibration control, Engineering Structures, 188 (2019) 480-492. 
https://doi.org/10.1016/j.engstruct.2019.03.030.

[38] Z.P. Zhao, Q.J. Chen, R.F. Zhang, C. Pan, Y.Y. Jiang, Optimal design of an inerter isolation system considering the soil condition, Engineering Structures, (2019). https://doi.org/10.1016/j.engstruct.2019.109324.

[39] Z.P. Zhao, R.F. Zhang, Y.Y. Jiang, C. Pan, Seismic response mitigation of structures with a friction pendulum inerter system, Engineering Structures, 193 (2019) 110-120. https://doi.org/10.1016/j.engstruct.2019.05.024.

[40] R.F. Zhang, Z.P. Zhao, C. Pan, Influence of mechanical layout of inerter systems on seismic mitigation of storage tanks, Soil Dynamics and Earthquake Engineering, (2018). https://doi.org/10.1016/j.soildyn.2018.07.036.

[41] C. Pan, R.F. Zhang, Design of structure with inerter system based on stochastic response mitigation ratio, Structural Control and Health Monitoring, 25 (2018) e2169. http://dx.doi.org/10.1002/stc.2169.

[42] K. Saito, Y. Sugimura, S. Nakaminami, H. Kida, N. Inoue, Vibration tests of 1 -story response control system using inertial mass and optimized softy spring and viscous element, in: the 14th World Conference on Earthquake Engineering 2008.

[43] SEAOC, Bluebook: The recommended lateral force requirements of the structural, SEAOC, 1959.

[44] K. Ikago, Y. Sugimura, K. Saito, N. Inoue, Simple design method for a tuned viscous mass damper seismic control system, in: the 15th World Conference on Earthquake Engineering, Lisbon, Portugal, 2012.

[45] N.M. Newmark, E. Rosenblueth, Fundamentals of earthquake engineering, Prentice-Hall, Englewood Cliffs, N.J., 1971.

[46] J. Lin, Y. Zhao, Y. Zhang, Accurate and highly efficient algorithms for structural stationary/non-stationary random responses, Computer Methods in Applied Mechanics \& Engineering, 191 (2001) 103-111.

[47] J.H. Lin, W.S. Zhang, F.W. Williams, Pseudo-excitation algorithm for nonstationary random seismic responses, Engineering Structures, 16 (1994) 270-276. http://dx.doi.org/10.1016/0141-0296(94)90067-1.

[48] Y.L. Xu, W.S. Zhang, J.M. Ko, J.H. Lin, Pseudo-excitation method for vibration analysis of wind-excited structures, Journal of Wind Engineering and Industrial Aerodynamics, 83 (1999) 443-454. http://dx.doi.org/10.1016/S01676105(99)00092-6.

[49] PEER, PEER Ground Motion Database, in, Pacific Earthquake Engineering Research Center, 2019. 